\title{
1 Linnemannia elongata (Mortierellaceae) stimulates Arabidopsis \\ 2 thaliana aerial growth and responses to auxin, ethylene, and reactive \\ 3 oxygen species
}

4

5 Natalie Vandepol ${ }^{1}$, Julian Liber ${ }^{2,3}$, Alan Yocca², Jason Matlock4, Patrick Edger ${ }^{5}$, Gregory Bonito ${ }^{1,6}$

6

$7 \quad{ }^{1}$ Michigan State University, Department of Microbiology \& Molecular Genetics, East Lansing MI

848825

$9 \quad{ }^{2}$ Michigan State University, Department of Plant Biology, East Lansing MI 48824

10 3Michigan Duke University, Department of Biology, Durham NC, 27708

11 4Michigan State University, Department of Entomology, East Lansing MI 48824

12 5Michigan State University, Department of Horticulture, East Lansing MI 48824

$13{ }^{6}$ Michigan State University, Department of Plant Soil and Microbial Sciences, East Lansing MI

$14 \quad 48824$ 


\section{Abstract}

Harnessing the plant microbiome has the potential to improve agricultural yields and protect plants against pathogens and/or abiotic stresses, while also relieving economic and environmental costs of crop production. While previous studies have gained valuable insights into the underlying genetics facilitating plant-fungal interactions, these have largely been skewed towards certain fungal clades (e.g. arbuscular mycorrhizal fungi). Several different phyla of fungi have been shown to positively impact plant growth rates, including Mortierellaceae fungi. However, the extent of the plant growth promotion (PGP) phenotype(s), their underlying mechanism(s), and the impact of bacterial endosymbionts on fungal-plant interactions remain poorly understood for Mortierellaceae. In this study, we focused on the symbiosis between soil fungus Linnemannia elongata (Mortierellaceae) and Arabidopsis thaliana (Brassicaceae), as both organisms have high-quality reference genomes and transcriptomes available, and their lifestyles and growth requirements are conducive to research conditions. Further, L. elongata can host bacterial endosymbionts related to Mollicutes and Burkholderia. The role of these endobacteria on facilitating fungal-plant associations, including potentially further promoting plant growth, remains completely unexplored. We measured Arabidopsis aerial growth at early and late life stages, seed production, and used mRNA sequencing to characterize differentially expressed plant genes in response to fungal inoculation with and without bacterial endosymbionts. We found that $L$. elongata improved aerial plant growth, seed mass and altered the plant transcriptome, including the upregulation of genes involved in plant hormones and "response to oxidative stress", "defense response to bacterium", and "defense response to fungus". Furthermore, the expression of genes in certain phytohormone biosynthetic pathways were found to be modified in plants treated with L. elongata. Notably, the presence of Mollicutes- or Burkholderia-related endosymbionts in 


\section{Introduction}

43 Microbial promotion of plant growth has great potential to improve agricultural yields and protect

44 plants against pathogens and/or abiotic stresses, while also relieving economic and

45 environmental costs of crop production [1,2]. Agriculturally important metrics pertaining to plant

46 growth promotion include aerial biomass, root biomass, root architecture, seed number, seed

47 size, and flowering time. Early-diverging filamentous fungi in the Mucoromycota are one group of

48 plant beneficial microbes, which have been hypothesized to have assisted plants in the

49 colonization of land [3]. There are three main guilds of plant mutualistic fungi relevant to this study:

50 arbuscular mycorrhizal (AM) fungi, ectomycorrhizal (EM) fungi, and non-mycorrhizal (NM)

51 endophytic fungi. For the purpose of this study, NM root endophytes are defined as fungi that are

52 found inside healthy plant roots but do not make characteristic mycorrhizal structures. Most of

53 these fungi are thought to promote plant growth primarily by providing water and mineral nutrients,

54 and sometimes secondarily by precluding infection by pathogens and/or priming and regulating

55 plant defense responses [4]. However, the signaling mechanisms and fungal symbiotic structures

56 are very distinct between and within these functional guilds, largely because most EM and NM

57 associations represent convergent evolution on a phenotype, rather than a shared evolutionary

58 mechanism of interaction [5].

59 Mortierellaceae are soil fungi in the subphylum Mortierellomycotina [6]. They are closely related

60 to Glomeromycotina (AMF) and Mucoromycotina, some of which are EM fungi $[3,7,8]$. Plant

61 associations with Mortierellaceae have been recorded since the early 1900s and these fungi are

62 broadly considered NM plant associates [9-11]. Many studies have investigated the impacts of

63 Mortierellaceae fungi on plant growth, however, the extent of the plant growth promotion (PGP)

64 phenotype(s) and the mechanism(s) underlying their association are still not well understood [12-

65 15].

66 Recent inoculation studies of Mortierellaceae on plant roots show that these fungi elicit a strong

67 PGP phenotype on a broad range of plant hosts [1,12,15]. Maize plants inoculated with

68 Linnemannia elongata (=Mortierella elongata) had increased plant height and dry aerial biomass

69 and analysis of phytohormone levels indicated high levels of abscisic acid and the auxin IAA

70 (indole-3-acetic acid) in response to L. elongata [1]. In contrast, Arabidopsis thaliana Col-0

71 (hereafter Arabidopsis) inoculated with L. hyalina (=Mortierella hyalina) also showed increased

72 total leaf surface area and aerial dry biomass, with reduced levels of abscisic acid and no

73 stimulation of auxin-responsive genes [12]. Mortierella antarctica was shown to increase the

74 growth of winter wheat by producing phytohormones IAA and gibberellic acid (GA) and the 
enzyme ACC (1-aminocyclopropane-1-carboxylate) deaminase, which degrades ACC, a

76 precursor to the phytohormone ethylene [15].

77 Recent studies have demonstrated Mortierellaceae can harbor endobacterial symbionts [16-19].

78 However, the impacts of endohyphal bacteria on the PGP phenotype have not been assessed.

79 Although the incidence of endobacteria within isolates of Mortierellaceae is quite low $(<10 \%)$, a

80 diversity of bacteria including Mycoavidus cysteinexigens and Mycoplasma-related endobacteria

81 (MRE) are known to colonize mycelium of diverse species across most of the genera in the family

82 [16-18,20]. Many species including L. elongata can harbor either Mycoavidus cysteinexigens or

83 MRE, however, there is generally a single lineage of endobacteria within any particular isolate

84 [16]. Both MRE and Ca. Glomeribacter, a Burkholderia-related endobacteria (BRE) that is

85 phylogenetically the sister group to Mycoavidus, are found in the Glomeromycotina. Ca.

86 Glomeribacter has been shown to increase fungal-host biological potential, and is hypothesized

87 to impact plant interactions as a mutualistic partner $[21,22]$.

88 In this study, we have focused on interaction between L. elongata and Arabidopsis, as both 89 organisms have reference genomes and transcriptomes available. Further, Arabidopsis is small, 90 has a short lifespan, and is ideal for follow-up studies relying on genetic manipulation. We used 91 two isolates of $L$. elongata, NVP64 and NVP80, to better understand mechanisms underlying $L$. 92 elongata symbiosis with plants. These two isolates of $L$. elongata were isolated from the same 93 soil, but were found to be colonized by different endobacteria; NVP64 contains Mycoavidus 94 cysteinexigens (BRE) while NVP80 contains MRE, designated as NVP64wt and NVP80wt given 95 that they are the wild-types of these strains. To determine whether either endobacterium has an 96 impact on the plant-fungal symbiosis we generated "cured" isogenic lines of each isolate, 97 NVP64cu and NVP80cu, where the endobacteria were removed through antibiotic passaging. We 98 hypothesized that $L$. elongata would provide a PGP phenotype and that endobacteria would 99 impact this response. We measured PGP of aerial growth at early and late life stages, seed 100 production, and used RNA sequencing to characterize differentially expressed plant genes in 101 response to fungal and endobacteria treatments. 


\section{Materials \& Methods}

\section{Plant and fungal culturing}

105 Growth media

106 Fungal strains were cultured in malt extract broth [MEB: $10 \mathrm{~g} / \mathrm{L}$ Malt Extract (VWR), $1 \mathrm{~g} / \mathrm{L}$ Bacto

107 Yeast Extract (Difco, Thomas Scientific; New Jersey, USA)], malt extract agar [MEA: 10 g/L Malt

108 Extract, $1 \mathrm{~g} / \mathrm{L}$ Bacto Yeast Extract, $10 \mathrm{~g} / \mathrm{L}$ Bacto Agar (Difco)], and Kaefer Medium [KM: $20 \mathrm{~g} / \mathrm{L}$

109 D-Glucose, $2 \mathrm{~g} / \mathrm{L}$ Peptone, $1 \mathrm{~g} / \mathrm{L}$ Yeast Extract, $1 \mathrm{~g} / \mathrm{L}$ Bacto Casamino Acids (Difco), $2 \mathrm{~mL} / \mathrm{L} \mathrm{Fe-}$ EDTA [2.5 $\mathrm{g} \mathrm{FeSO}_{4}{ }^{*} 7 \mathrm{H}_{2} \mathrm{O}, 3.36 \mathrm{~g} \mathrm{Na}_{2}$ EDTA, $500 \mathrm{~mL}$ water], $50 \mathrm{~mL} / \mathrm{L} \mathrm{KM}$ Macronutrients [12 g/L $\mathrm{NaNO}_{3}, 10.4 \mathrm{~g} / \mathrm{L} \mathrm{KCl}, 10.4 \mathrm{~g} / \mathrm{L} \mathrm{MgSO}{ }_{4}{ }^{*} 7 \mathrm{H}_{2} \mathrm{O}, 30.4 \mathrm{~g} / \mathrm{L} \mathrm{KH}_{2} \mathrm{PO}_{4}$ ], $10 \mathrm{~mL} / \mathrm{L} \mathrm{KM}$ Micronutrients [2.2 $\mathrm{g} / \mathrm{L} \mathrm{ZnSO}{ }_{4}{ }^{*} 7 \mathrm{H}_{2} \mathrm{O}, 2.2 \mathrm{~g} / \mathrm{L} \mathrm{H}_{3} \mathrm{BO}_{3}, 0.16 \mathrm{~g} / \mathrm{L} \quad \mathrm{CuSO}_{4}{ }^{*} 5 \mathrm{H}_{2} \mathrm{O}, 0.5 \mathrm{~g} / \mathrm{L} \quad \mathrm{MnSO}_{4}{ }^{*} \mathrm{H}_{2} \mathrm{O}, 0.16 \mathrm{~g} / \mathrm{L}$ $\left.\mathrm{CoCl}_{2}{ }^{*} 5 \mathrm{H}_{2} \mathrm{O}, 0.11 \mathrm{~g} / \mathrm{L}\left(\mathrm{NH}_{4}\right)_{6} \mathrm{Mo}_{7} \mathrm{O}_{24}{ }^{*} 4 \mathrm{H}_{2} \mathrm{O}\right], \mathrm{pH} 6.5$ with $10 \mathrm{~N} \mathrm{KOH}$, and supplemented with

114 Thiamine $(1 \mathrm{mg} / \mathrm{L})$ and Biotin $(0.5 \mathrm{mg} / \mathrm{L})$ after autoclaving and cooling to $60^{\circ} \mathrm{C}$ ]. Sterilized seeds were germinated on Murashige \& Skoog (MS) medium [1xMS: $4.4 \mathrm{~g} / \mathrm{L}$ Murashige and Skoog medium (Sigma Aldrich; Missouri, USA), pH $5.7 \mathrm{w} / \mathrm{KOH}$, and $10 \mathrm{~g} / \mathrm{L}$ agar (Sigma, produc\# A1296)]. Plant-fungal experiments were conducted on Plant Nutrient Medium [PNM: $0.5 \mathrm{~g} / \mathrm{L}$ $\mathrm{KNO}_{3}, 0.49 \mathrm{~g} / \mathrm{L} \mathrm{MgSO}{ }_{4}{ }^{*} 7 \mathrm{H}_{2} \mathrm{O}, 0.47 \mathrm{~g} / \mathrm{L} \mathrm{Ca}\left(\mathrm{NO}_{3}\right)_{2}{ }^{*} 4 \mathrm{H}_{2} \mathrm{O}, 2.5 \mathrm{~mL} / \mathrm{L}$ Fe-EDTA, $1 \mathrm{~mL} / \mathrm{L}$ PNM Micronutrients [4.3 g/L Boric Acid, $2.8 \mathrm{~g} / \mathrm{L} \mathrm{MnCl}{ }_{2}{ }^{*} 4 \mathrm{H}_{2} \mathrm{O}, 124.8 \mathrm{mg} / \mathrm{L} \mathrm{CuSO}{ }_{4}{ }^{*} 5 \mathrm{H}_{2} \mathrm{O}, 287.5 \mathrm{mg} / \mathrm{L}$ $\mathrm{ZnSO}_{4}{ }^{*} 7 \mathrm{H}_{2} \mathrm{O}, 48.4 \mathrm{mg} / \mathrm{L} \mathrm{Na}_{2} \mathrm{MoO}_{4}{ }^{*} 2 \mathrm{H}_{2} \mathrm{O}, 2.4 \mathrm{mg} / \mathrm{L} \mathrm{CoCl}{ }^{*} 6 \mathrm{H}_{2} \mathrm{O}$ ], $10 \mathrm{~g} / \mathrm{L}$ agar (Sigma, product A1296), autoclaved and the $\mathrm{pH}$ adjusted with $2.5 \mathrm{~mL} / \mathrm{L} 1 \mathrm{M} \mathrm{H}_{2} \mathrm{KPO}_{4}$ before pouring 22-24mL per $100 \mathrm{~mm}$ square plate (with grid)].

123 To generate a fungal substrate suitable for inoculating potting mix, white millet (Natures Window;

124 Michigan, USA), horticultural perlite (PVP Industries, Inc; Ohio, USA), and pearled barley 125 (International Foodsource; New Jersey, USA) were each soaked overnight in DI water. The millet 126 and barley were each boiled in fresh DI water on a hotplate until the grains began to break open, 127 then removed from the hotplate and drained of excess water. When prepared, millet and barley 128 expand to about $150 \%$ and $300 \%$ of the dry volume, respectively. The boiled millet, boiled barley, 129 and perlite were mixed in a 2:1:1 v:v:v ratio. For each treatment, $600 \mathrm{~mL}$ of this "millet mix" was 130 placed into a gusseted Unicorn bag (Unicorn Bags, type 10T; Texas, USA) and autoclaved for 45 131 minutes, allowed to rest overnight under a sterile hood and autoclaved again for 45 minutes.

132 To generate sterile SureMix-based plant growth substrates, SureMix Perlite (Michigan Growers 133 Products; Michigan, USA) substrate was saturated with deionized water, which was measured 134 and placed into autoclavable bags to ensure the correct volume would be available. A single bag 
was used for each experimental treatment. The bags of SureMix substrate were autoclaved 45 minutes on a liquid cycle, stored at room temperature for 3-7 days, autoclaved again for 45 minutes on a liquid cycle, cooled to room temperature, and rinsed through with $3 \mathrm{~L}$ of sterile MilliQ water $(18 \mathrm{M} \Omega \cdot \mathrm{cm})$. The autoclaved SureMix was rinsed to remove autoclaving byproducts by flushing with $3 \mathrm{~L}$ of sterile MilliQ water on a dish cart covered with a double layer of window screen mesh which had been sterilized with bleach and rinsed with autoclaved MilliQ water.

\section{Curing fungi of endobacteria}

142 Replicated lines of $L$. elongata NVP64wt and NVP80wt were cured of their endobacteria by 143 repeated culturing in media containing antibiotics, a protocol adapted from Uehling et al. (2017).

144 Fungi were transferred between MEB and MEA supplemented with $1 \mathrm{~g} / \mathrm{L}$ Bacto Peptone (Difco), $145100 \mu \mathrm{g} / \mathrm{mL}$ ciprofloxacin, $50 \mu \mathrm{g} / \mathrm{mL}$ kanamycin, $50 \mu \mathrm{g} / \mathrm{mL}$ streptomycin, and $50 \mu \mathrm{g} / \mathrm{mL}$ chloramphenicol. Each transfer was performed by transplanting a 1-4 $\mathrm{mm}^{2}$ piece of tissue from the outer edge or surface of the mycelium with a Nichrome inoculating loop and submerging the tissue under the agar surface or broth to maximize contact of the growing hyphae with the antibiotics. Transfers were performed every 3 or 4 days, alternating agar and broth substrate, for 6 transfers.

Following antibiotic curing, tissue from the original and newly-cured lines, as well as the wild-type line, were cultured on antibiotic-free $60 \mathrm{~mm}$ MEA plates with an autoclaved cellophane sheet placed atop the agar. After 13 days of incubation, fungal tissue was scraped off the cellophane and DNA extracted using a CTAB-based chloroform extraction protocol (Supplementary Materials and Methods [23].

\section{Arabidopsis seed sterilization \& germination}

157 Arabidopsis thaliana Col-0 CS70000 were obtained from the Arabidopsis Biological Resource 158 Center. Seeds were germinated and grown for three generations in a grow room. Bulk seed was 159 collected from the third generation and screened to homogenize seed size with $350 \mu \mathrm{m}$ and 250 $160 \mu \mathrm{m}$ sieves (VWR, Pennsylvania, USA), retaining the middle fraction.

161 Arabidopsis seeds were divided from the screened stock into $1.5 \mathrm{~mL}$ Eppendorf tubes using a 162200 seed spoon, with up to 1200 seeds per tube. Seeds were surface sterilized by washing in $163800 \mu \mathrm{L} \mathrm{70 \%} \mathrm{Ethanol} \mathrm{for} 20$ seconds, discarding the ethanol, and then washing in $400 \mu \mathrm{L} 20 \%$ 164 bleach (Clorox Performance, 8.3\% Sodium Hypochlorite, Clorox, California, USA) for 30 seconds. 165 Seeds were then rinsed of bleach three times by quenching with $1 \mathrm{~mL}$ sterile water and discarding the liquid. Seeds were then resuspended in $500 \mu \mathrm{L}$ sterile water prior to planting. 
167 Surface sterilized seeds were plated on 1XMS using a p1000 and sterile water, 16 seeds per plate

168 in rows of 3, 4, 5, and 4, with about $1 \mathrm{~cm}$ between seeds and rows (Supplementary Fig. S1a).

169 We germinated at least 5 times as many seeds as were needed for the experiment to allow greater

170 control of seedling size.

171 Germination 1xMS plates were cold stratified for 2 days in the dark at $4^{\circ} \mathrm{C}$ to synchronize

172 germination, then allowed to germinate and grow for 5 or 10 days, depending on the experiment,

173 in a Percival I-36LLVL growth chamber at 103-118 $\mu \mathrm{mol} / \mathrm{m}^{2} \bullet$ S PAR with $16 \mathrm{hr}$ day \& $8 \mathrm{hr}$ night,

$17422^{\circ} \mathrm{C}$, ambient humidity. Light levels were measured using an LI-250A light meter (LI-COR,

175 Nebraska, USA).

\section{Potting mix experiments}

\section{Grain-based inoculum}

178 Each fungal strain was grown in $250 \mathrm{~mL}$ Erlenmeyer flasks with $75 \mathrm{~mL}$ of MEB for 2 weeks.

179 Colonized medium was poured out into an autoclaved beaker and the mycelium collected with

180 sterile tweezers, coarsely chopped in a sterile petri dish, and added to sterile millet mix bags. The 181 bags were lightly mixed, sealed in two places with an impulse sealer, and the fungi allowed to 182 colonize the spawn for two weeks.

183 Arabidopsis growth conditions

184 Five days after germination, Arabidopsis seedlings were transplanted from 1xMS plates to plug 185 trays of autoclaved and rinsed SureMix and moved to a Bio Chambers AC-40 growth chamber 186 with $16 \mathrm{hr}$ day, $8 \mathrm{hr}$ night, $22^{\circ} \mathrm{C}$, ambient humidity. Seedlings were grown in plugs for 11 days (16 187 days after germination). The soil plugs and seedling roots were treated with Zerotol 2.0 (BioSafe 188 Systems, Connecticut, USA), an algaecide, bactericide, and fungicide containing Hydrogen 189 Peroxide \& Peroxyacetic Acid. The Zerotol was applied as a soil drench for 15 minutes, rinsed 190 three times with distilled water, and transplanted into $4 \mathrm{in}^{3}$ pots with SureMix mixed with the 191 appropriate millet mix treatment. Each treatment was contained in a separate waterproof tray with 192 an 18 pot capacity (3 rows of 6 pots). Using seventeen pots per treatment left an empty spot for 193 watering. Four days after transplanting, seedlings were treated with 2 L of Peters 20-20-20 194 fertilizer mixed at $1 / 8$ th strength $(0.1 \mathrm{tsp} / \mathrm{L})$ in MilliQ water. Thereafter, plants were watered with 195 MilliQ water as needed. 


\section{Above ground biomass}

197 At 34 days after transplanting and inoculation (50 days after germination), all treatments were 198 observed to have ripening siliques, necessitating harvesting to avoid excessive loss of seed 199 biomass during plant handling. Twelve plants per treatment were harvested by cutting the roots 200 at the potting mix line and trimming and/or folding the aerial parts into tared envelopes (Top Flight no.10 Security Envelope, Strip \& Seal). Fresh weight was recorded immediately after harvesting was complete. Plants were dried at room temperature $\left(20-22^{\circ} \mathrm{C}\right)$ for 2 weeks and re-weighed for the dry biomass. All envelope and plant biomass measurements were taken on a Mettler Toledo PG2002-S scale.

Seed collection

206 Five plants were randomly selected for seed collection. ARACON tubes (Arasystem, Belgium) 207 were installed over the rosette. When the remaining plants were harvested for biomass, these 208 five plants were moved to a drying room for two weeks. Dry plant material was collected and stored in wax paper bags until processing. Seeds were isolated from plant material by manually massaging the bags to release seeds, filtering through a Rösle Stainless Steel Fine Mesh Tea Strainer (Wire Handle, 3.2-inch, model\# 95158) to remove large plant debris, repeatedly passing

212 over copier paper, and picking out remnant plant matter with tweezers. Cleaned seeds were 213 collected in tared $2 \mathrm{~mL}$ Eppendorf tubes and weighed on a Mettler Toledo AB104-S/FACT scale. 214 To determine average seed mass, approximately $14 \mathrm{mg}$ of seeds per sample were weighed on 215 an ultrasensitive balance, adhered to a piece of white paper using a glue stick, covered by clear 216 packing tape, scanned, and counted by image analysis in ImageJ following protocols optimized 217 by Dr. Mathew Greishop's lab, based on the work of Mark Ledebuhr (Supplementary Materials 218 and Methods, Supplementary Fig. S2).

\section{Statistical analysis}

220 Since the data were non-normal, we performed Wilcox ranked sum tests and adjusted p-values 221 for multiple comparisons using the Holm method. Between NVP64cu v. NVP64wt, NVP80cu v. 222 NVP80wt, and NoMillet v. Uninoculated, we used two-tailed tests. Between each fungal treatment 223 and the Uninoculated, we performed one-tailed tests with the alternative hypothesis being "less" 224 or "greater" as appropriate. Data analysis and visualization was conducted in R using the ggpubr 225 and ggsignif packages [24,25]. Datasets and code are available at https://github.com/natalie226 vandepol/Arabidopsis-L.elongata-PGP. 


\section{Agar-based experiments}

\section{Transplanting \& inoculation}

229 We based the design of these experiments on the methodology used by Johnson et al.[12]. Arabidopsis seeds were surface sterilized and germinated as described previously. Ten days after germination, seedlings were categorized into three approximate seedling size "categories": too small, too big, and average. Three "average" seedlings were transplanted to each PNM plate such that the cotyledons aligned with the top line of the plate grid and the roots were not covered by

234 the grid pattern (Supplementary Fig. S1b). Each plate was numbered as it was populated with seedlings so that plates could be assigned to treatments serially (e.g., 1-A, 2-B, 3-C, 4-A, 5-B, $6-\mathrm{C}, 7-\mathrm{A}$, etc.), to homogenize variation and bias in seedling size throughout the transplant procedure. Plates were inoculated by transferring two $5 \mathrm{~mm} \times 5 \mathrm{~mm}$ squares of Kaefer Medium, sterile or colonized by the appropriate fungal culture, between the three seedlings.

\section{Root length}

240 After transplanting and inoculation, seedlings and fungi were left undisturbed overnight to allow 241 them to adhere to the media and minimize the likelihood of movement during handling. The

242 following day (1 day post inoculation), plates were imaged on an Epson scanner at 1200 dots per 243 inch using Home mode and default settings (Supplementary Fig. S1b). Images were processed 244 in ImageJ v.1.52p, using the $13 \mathrm{~mm}$ grid on the plates as a scale, the freehand line tool to trace 245 the roots, and the measuring tool to determine starting root length of each seedling.

\section{Growth chamber}

247 Light levels were measured with a LI-250A light meter (LI-COR) at 9 different points on each of 248 the four shelves in the growth chamber (Supplementary Table S1). To homogenize variability in 249 environmental conditions across treatments, plates were distributed between light level regions 250 and the lower three shelves as evenly as possible and their location in the chamber recorded. 251 Each of the shelves accommodate 3 rows of 15 plates, with 5 plates assigned to each of the 9 252 zones on the shelf (Supplementary Fig. S3).

\section{Bolting panel}

254 To determine whether bolting time was affected by fungal colonization, PNM plates with 10 day 255 old Arabidopsis seedlings were inoculated and monitored daily for evidence of bolting, which was 256 defined as visible elongation of the emerging inflorescence away from the rosette 257 (Supplementary Fig. S4). As each plant bolted, the date was noted on the plate. 
Harvesting aerial plant material

259 At 12 DPI (22 days old), the aerial portion of each plant was cut away from the roots and placed 260 into a folded "envelope" made from weighing paper and dried in a $65^{\circ} \mathrm{C}$ drying oven for 48 hours.

261 The envelopes of dried plants were stored in empty tip boxes and double bagged with Ziplock 262 bags to prevent reabsorption of atmospheric water before weighing. Dry plants were weighed on 263 a DeltaRange XP26 ultrasensitive balance (Mettler Toledo; Ohio, USA).

\section{Statistical analysis}

265 We conducted statistical analyses in R v.3.6.0 using the tidyverse v1.3.0, Ime4 v1.1-21, ImerTest 266 v3.1-1, car v3.0-6 packages [26-30]. Bolting data were visualized as boxplots and visibly non267 normal. We used the Kruskal-Wallis test to examine differences in bolting age between treatments 268 [31].

269 Aerial dry weight data were visualized as boxplots and assessed as approximately normal and 270 homoskedastic. We used analysis of variance (ANOVA) and linear models to examine differences 271 in dry weight within each experimental dataset to determine the effects of environmental factors 272 tested by each experiment. Based on the results of these tests, we constructed a linear mixed 273 model of the combined dry weight data from the two agar experiments, specifying treatment and 274 seedling root length as fixed effects and experiment (Media Panel \& Cured Panel) and plate (to 275 account for three plants measured per plate) as grouping factors:

$$
\text { DryWeight } \sim \text { Treatment + RootLength + (1 | Experiment : Plate })
$$

277 We used the emmeans v1.4.4 package to perform pairwise comparisons of the model estimates 278 for each treatment [32]. The estimated marginal mean, confidence interval, and significance 279 groups were extracted for graphical summarization.

280 Root microscopy

281 Seedlings were grown on MS plates (see above) for five days post-stratification, then transferred 282 to PNM plates, with four seedlings per plate, approximately $1 \mathrm{~cm}$ from the plate edge. Two $\sim 5 \mathrm{~mm}$ $283 \times 10 \mathrm{~mm}$ blocks of MEA colonized with mycelium of L. elongata NVP64cu or NVP80cu were 284 placed on the centerline of the plate, spaced between plants 1 and 2 then 3 and 4 . The plates 285 were sealed with parafilm and arranged vertically in a Percival I-36LLVL growth chamber at 75 $286 \mu \mathrm{mol} / \mathrm{m}^{2} \bullet \mathrm{s}$ PAR with $16 \mathrm{hr}$ day $\& 8 \mathrm{hr}$ night, $22^{\circ} \mathrm{C}, 60 \%$ relative humidity. Roots were sampled at $28713 \mathrm{DPI}$ (18 days old) and again at 23-25 DPI (28-30 days old). 
Roots were cut from shoots using a scalpel, then forceps were used to transfer roots to $200 \mu \mathrm{L}$ of stain solution or $1 \mathrm{x}$ PBS, $\mathrm{pH} 7.2$, in a $500 \mu \mathrm{L}$ conical tube. The stain solution was composed of $25 \mu \mathrm{g} / \mathrm{mL}$ WGA-640R (Biotium, California, USA) and $1 \mathrm{mg} / \mathrm{mL}$ calcofluor white M2R, in 1x PBS, $\mathrm{pH}$ 7.2. Vacuum was applied to the roots in liquid with a vacuum pump, three times for $30 \mathrm{~s}$ each, releasing the pressure after each time. The roots in stain or buffer were incubated at room temperature for $30 \mathrm{~min}$ on a table shaker at $60 \mathrm{rpm}$.

294 Roots were removed from the stain solution and placed on glass slides, then coverslips were 295 added. Roots were imaged using an Olympus Fluoview FV10i confocal laser scanning 296 microscope. The unstained roots were viewed first and used to calibrate sensitivity values. The 297 WGA-640R channel was viewed with $\lambda_{\mathrm{ex}} \sim 642 \mathrm{~nm}, \lambda_{\mathrm{em}} \sim 661 \mathrm{~nm}$ and the calcofluor white M2R 298 channel was viewed with $\lambda_{\mathrm{ex}} \sim 352 \mathrm{~nm}, \lambda_{\mathrm{em}} \sim 455 \mathrm{~nm}$. The micrographs were processed, recolored, and transformed in ImageJ v1.53h [33] and 3D Slicer v4.11.20210226 [34].

\section{RNA sequencing \& differential gene expression}

\section{$301 \quad$ Root harvesting \& storage}

302 The root material for the RNAseq experiment was collected from the plants generated in Agar experiments (Fig. 1, see Agar-based experiments: Harvesting aerial plant material Methods section). Before collecting the aerial parts of the plants for biomass assays, five plates were selected from each treatment on the basis of similar light levels within the chamber. For each selected plate, two RNAse-zap treated, DEPC water rinsed, autoclaved steel beads were placed in one RNAse-free $1.5 \mathrm{~mL}$ Eppendorf tube, handled with gloves treated with RNAse-zap. Eppendorf tubes were placed in an autoclavable tube box, open and upright, the box wrapped in

309 foil and autoclaved for 25 minutes on a dry cycle. After autoclaving, wearing RNAse-zap treated 310 gloves, the Eppendorf tubes were carefully removed from the box, closed, and labeled with the 311 numbers of the plates from which the roots were to be collected.

312 During harvest, each selected plate was removed individually from the chamber, opened, and the 313 roots collected with forceps and a scalpel. The roots were immediately placed in a cold Eppendorf 314 tube and flash frozen in liquid nitrogen. The time between removing the plate from its place in the 315 chamber to freezing the Eppendorf tube and roots did not exceed 30 seconds. The forceps and 316 scalpel were soaked in $10 \%$ bleach between samples and excess liquid wicked off by a paper 317 towel before contacting the roots. The Eppendorf tubes of root samples were stored at $-80^{\circ} \mathrm{C}$ prior to extracting RNA. 


\section{RNA extraction}

320 Tissue was homogenized by three 30 second bursts at $30 \mathrm{~Hz}$ in a TissueLyzer II (Qiagen; 321 Germany), with 30 second rests in liquid nitrogen between each burst. RNA was extracted using 322 a Qiagen RNEasy Plant Mini Kit, employing $450 \mu \mathrm{L}$ Buffer RLT lysis buffer (with $10 \mu \mathrm{L} \beta-\mathrm{ME}$ per $3231 \mathrm{~mL}$ Buffer RLT), an on-column DNAse digest (RNase-Free DNase Set, Qiagen), and eluting 2x 324 with $50 \mu \mathrm{L}$ RNAse free water. A $5 \mu \mathrm{L}$ aliquot was set aside to perform an initial quantification using 325 a NanoDrop. Samples with less than $75 \mu \mathrm{g} / \mathrm{mL}$ were concentrated by ethanol precipitation as described below. RNA was quantified and quality checked using BioAnalyzer (MSU RTSF). All RNA samples had RIN scores $>9.0$.

329 Low concentration RNA extractions were amended with $10 \mu \mathrm{L} 3 \mathrm{M}$ Sodium Acetate and then 300

$330 \mu \mathrm{L}$ ice cold $100 \%$ ethanol, vortexed briefly to mix, and precipitated at $-20^{\circ} \mathrm{C}$ overnight. RNA was 331 pelleted by centrifuging for $30 \mathrm{~min}$ at full speed at $4^{\circ} \mathrm{C}$. The RNA pellet was washed with $200 \mu \mathrm{L}$ 332 ice cold $70 \% \mathrm{EtOH}$ and centrifuged for $10 \mathrm{~min}$ at full speed at $4^{\circ} \mathrm{C}$. The supernatant was discarded 333 and the pellet air-dried for $15 \mathrm{~min}$ on the bench before being resuspended in 30-50 $\mu \mathrm{L}$ RNAse334 free water. A $5 \mu \mathrm{L}$ aliquot was taken for quantity and quality analysis and the remainder stored at $335-80^{\circ} \mathrm{C}$.

\section{$336 \quad$ Library preparation \& sequencing}

337 Libraries were prepared using the Illumina TruSeq Stranded mRNA Library Preparation Kit with 338 the IDT for Illumina Unique Dual Index adapters following manufacturer's recommendations. 339 Completed libraries were QC'd and quantified using a combination of Qubit dsDNA HS and 340 Agilent 4200 TapeStation High Sensitivity DNA 1000 assays. The libraries were pooled in 341 equimolar amounts and the pool quantified using the Kapa Biosystems Illumina Library 342 Quantification qPCR kit. This pool was loaded onto an Illumina NextSeq 500/550 High Output 343 flow cell (v2.5) and sequencing performed in a 1x75 bp single read format using a NextSeq $344500 / 550$ High Output 75 cycle reagent kit (v2.5). Base calling was done by Illumina Real Time 345 Analysis (RTA) v2.4.11 and output of RTA was demultiplexed and converted to FastQ format with 346 Illumina Bcl2fastq v2.19.1. Sequence data have been accessioned in NCBI's SRA under the 347 BioProject PRJNA704083.

$348 \underline{\text { qPCR }}$

349 Primer sets for qPCR were designed using 16S rRNA gene sequences of $L$. elongata NVP64 and 350 NVP80 endobacteria with the IDT PrimerQuest $\AA$ Tool for 2 primers and intercalating dye 351 (Supplementary Table S2). Primer sets were verified using wild-type DNA samples, for which a 
standard curve was created with dilutions from 100 to $10-4$ and efficiencies were within $90-110 \%$. Absolute copy number calibration was not performed because only presence/absence validation was required. cDNA was synthesized for qPCR quantification using the LunaScript RT SuperMix Kit (New England Biolabs; Massachusetts, USA). qPCR reactions were composed of $7.5 \mu \mathrm{L}$ Power SYBR Green PCR Master Mix (ThermoFisher Scientific; Massachusetts, USA), $5.5 \mu \mathrm{L}$ nuclease-free water, $0.25 \mu \mathrm{L}$ each primer, and $1.5 \mu \mathrm{L}$ of undiluted template. The reaction cycle was $95^{\circ} \mathrm{C}$ for $10 \mathrm{~min}$, followed by 40 cycles of $95^{\circ} \mathrm{C}$ for $15 \mathrm{sec}$ and $60^{\circ} \mathrm{C}$ for 1 min with a fluorescence measurement. A melting curve was performed following amplification: $95^{\circ} \mathrm{C}$ for 15 $\mathrm{sec}$ and $60^{\circ} \mathrm{C}$ for $15 \mathrm{sec}$, then a $20 \mathrm{~min}$ ramp up to $95^{\circ} \mathrm{C}$, followed by $95^{\circ} \mathrm{C}$ for $15 \mathrm{sec}$. At least two reactions were performed per sample and primer combination.

\section{Sequence analysis}

Raw, demultiplexed reads were quality trimmed and filtered using Trimmomatic v.0.38 [35]. A combined reference transcriptome was constructed from the Arabidopsis Thaliana Reference Transcript Dataset 2 (AtRTD2_19April2016.fa, accessed 10/21/2019) and Linnemannia elongata AG77 (Morel2_GeneCatalog_transcripts_ 20151120.nt.fasta.gz, project 1006314, accessed 10/21/2019) [13,17]. This combined reference transcriptome was indexed in Salmon v0.11.3 and used to quasi-map the trimmed reads to transcripts [36].

\section{Differential gene expression analysis}

A transcript-to-gene (tx2gene) table was constructed in $R$ v.3.6.0 for Arabidopsis gene annotations (AtRTD2_19April2016.gtf.txt, accessed on 01/12/2020). Fungal reads were extremely rare in the dataset, thus, analyses focused solely on plant transcriptional responses $[26,37]$. Salmon quants.sf files were imported into R using tximport (type=salmon; Soneson et al. 2015). Differential gene expression analysis was carried out with both the EdgeR package and the DESeq2 package in $\mathrm{R}$ [38-40]. Gene expression was computed for each treatment across the three biological replicates, with the control treatment specified as the reference level in the experimental design matrix. Differentially expressed genes were identified by contrasting each fungal treatment against the control. In DESeq2, gene lists from each comparison were filtered by an adjusted p-value of 0.05 and an absolute value of log2 fold change (LFC) cutoff of 0.585 , which corresponds to a fold change in expression of 1.5. We generated volcano plots of these pairwise comparisons using the EnhancedVolcano package in $R$ [41]. In EdgeR, the gene list encompassed all four fungal treatments with a single $p$-value for each gene, so the EdgeR gene list was filtered by overall $p$-value and whether at least one fungal treatment LFC meeting the LFC cutoff $[38,39]$. The DESeq2 gene list was filtered to include only genes also present in the EdgeR 
gene list. Since DESeq2 provided p-values for each comparison, we used the log2-fold change and adjusted p-value of the DESeq2 analyses to compose our final DEG table. Gene ontology was assigned by referencing TAIR and UniProtKB annotation databases and synthesizing the most detailed and supported annotations $[42,43]$.

\section{Functional enrichment}

390 We generated a list of differentially expressed genes in response to at least a single fungal treatment. The list of up and down regulated genes were separately searched for functional enrichment using the clusterprofiler package in R. Code to reproduce the GO enrichment is publically available: https://github.com/Aeyocca/00_Collab/tree/main/plant_fungal_interactions (last accessed 11-03-2021) [44].

\section{Results}

\section{Potting Mix Experiments}

All fungal treatments had significantly higher aerial dry biomass than the uninoculated millet control. Aerial dry biomass of full-grown Arabidopsis plants was not significantly different between NVP64cu and NVP64wt or between NVP80cu and NVP80wt (Fig. 2). Millet has previously been used as a fungal substrate for inoculating soil in plant-fungal symbiosis research [13,45]. A NoMillet control was initially included to test the assumption that the millet-based inoculum had no impact on the plants. However, the NoMillet controls had the highest aerial dry biomass of any experimental treatment, indicating that the millet carrier negatively impacts plant health. Thus, results of the potting mix experiments may be interpreted in terms of stress mitigation. In this case, the NoMillet control presents a baseline and the Uninoculated control is an unmitigated

407 stress imposed by the millet grain. The fungal treatments generally fell between these two 408 treatments, indicating partial mitigation of the stress imposed by the grain-based inoculum. Given 409 that the exact nature of the stress imposed by the grain-based inoculum is unknown, we focused 410 our analyses of these data on fungal treatments $v$. uninoculated control and relied on pure culture 411 agar plate methods for subsequent experiments.

\section{Linnemannia elongata impacted Arabidopsis seed production}

413 As with the aerial biomass, the total seed mass of NVP80cu and NVP80wt were significantly 414 higher than the Uninoculated control (Fig. 3Aa). No significant differences in total or average seed 415 mass were found between the isogenic isolate pairs, i.e. NVP64wt vs. NVP64cu and NVP80wt 
vs. NVP80cu (Fig. 3). Unlike total seed mass, the average seed mass of the Uninoculated control was slightly higher than NVP80wt and NVP64cu, but not significantly different from the NoMillet control (Fig. 3b). The total seed mass in the NoMillet control was significantly higher than that of the Uninoculated control.

420 Given the potential that sufficient seeds in the fungal treatments could be smaller due to 421 incomplete development, rather than total reduction in seed size, we set out to determine whether 422 this might be visible in violin plots of individual seed pixel areas from the image analysis. This 423 would be represented by a bimodal violin with peaks representing immature and mature seeds. 424 No strong bimodality could be seen in replicates or treatments (Supplementary Fig. S5).

\section{Agar Experiments}

\section{Linnemannia elongata did not impact the timing of Arabidopsis bolting}

427 The Kruskal-Wallis Test was conducted to examine the age at which plants bolted according to

428 fungal treatment. With 27 plates per treatment and 3 plates per plate, no significant differences in 429 bolting time $(H=4.92, p=0.296, \mathrm{df}=4)$ were found between the five treatments. The mean age at 430 which an inflorescence could first be seen to elongate from the rosette was 22 days old, which 431 was 12 days post transplanting and inoculation (DPI). Therefore, we harvested all further agar 432 experiments at $12 \mathrm{DPI}$ to prevent bolting from affecting dry weight data, which differed from the 7 433 day co-cultivation time used by Johnson et al. (2019) (Fig. 1).

\section{Linnemannia elongata increased young Arabidopsis aerial dry biomass}

435 We expected that several environmental factors could potentially impact our observation of how 436 Arabidopsis responds to $L$. elongata. These included the (1) starting size of the plant; (2) local 437 light level, (3) medium on which the fungus was cultured, and (4) process by which the fungi were 438 cured of their endobacteria. We determined that there was no statistically significant correlation 439 between light level and harvested plant dry weight in any of the treatments (Supplementary 440 Table S3). We performed linear modeling of the dry weights as a function of medium, treatment, 441 and interaction between those, and determined there were no significant differences in harvested 442 plant dry weight based on media $\left(F_{1,110}=0.966, p=0.328\right.$; Supplementary Table S4) and no 443 significant interaction between medium and treatment $\left(F_{4,110}=0.331, p=0.857\right)$. Analysis of 444 variance found no statistically significant differences in mean harvested plant dry weight, between 445 three independently generated cured lines (L0, L1, and L2) of L. elongata, for both NVP64 $446\left(F_{2,42}=0.443 p=0.645\right)$ and NVP80 $\left(F_{2,42}=1.966, p=0.153\right)$, indicating that differences between 447 wild-type (wt) and cured (cu) strains are likely due to the presence/absence of endobacteria, 
rather than accumulated mutations from the antibiotic passaging protocol. Analysis of variance in seedling root length showed that the mean seedling root length was consistent between treatments of each experiment $\left(\mathrm{F}_{4,755}=0.953, p=0.433\right)$, but differed significantly between experiments $\left(F_{1,755}=267.3, p=2 \mathrm{e}-16\right)$, with no significant interaction effect $\left(F_{4,755}=0.541, p=0.706\right)$. Preliminary linear model analysis showed a significant positive correlation between seedling root length and harvested plant dry weight, with no significant differences between the slope of this correlation across experiments or treatments (Supplementary Table S5). We fit a linear mixed model of combined aerial dry weight data from both experiments as a function of treatment and seedling root length. Results of this model can be seen in Table 1. The estimated marginal means of aerial dry weight was significantly higher in all four fungal treatments compared to the control, but there were no significant differences between fungal treatments (Fig. 4).

\section{All Linnemannia elongata strains colonize Arabidopsis roots evenly}

460 We used the cycle number at which the fluorescent signal of the qPCR probe exceeded the threshold level to calculate the ratio of $L$. elongata RNA to Arabidopsis RNA in each reaction. This ratio represents the degree of fungal colonization of plant roots. There were no significant differences in this ratio between any of the fungal treatments $(p>0.1)$ and each lineage of endobacteria was detected only in the wild-type strains

(Supplementary Table S6; Supplementary Fig. S6).

466 We visually assessed the ability of $L$. elongata NVP64cu and NVP80cu to grow on and into root 467 tissue, and the localization of hyphae within the roots for plants grown on agar. At 13 DPI L. 468 elongata had colonized the root rhizosphere, but no internal hyphae were observed for NVP80cu. 469 However, by 23 DPI we observed NVP80cu hyphae within epidermal root cells and root hair cells, 470 with clearly visible plant cell walls bounding the hyphae on all sides (Fig 5a-f). Similarly, we 471 visualized NVP64cu growing to high density within epidermal cells at $25 \mathrm{DPI}$, and the mass of 472 hyphae bounded by the plant cell wall (Fig $5 \mathbf{g}-\mathbf{I})$.

\section{Differential Gene Expression}

\section{$474 \quad$ Molecular results}

475 We generated a total of 521.2 million sequence reads $(39.1 \mathrm{~Gb})$ at an average of 34.7 million 476 (30.5-37.8M) sequence reads per sample, with an average of 97.64\% (97.22-97.85\%) mapping 477 rate to the combined reference transcriptome. Of the mapped reads, an average of $99.82 \%$ 478 (98.64-99.99\%) mapped to plant transcripts (Supplementary Table S7). Thus, analyses were 479 focused on plant responses to experimental treatments. 
480 Arabidopsis differentially expressed genes in response to Linnemannia elongata

481 We conducted initial RNAseq data exploration in DESeq2 to confirm consistent gene expression

482 profiles between biological replicates of each condition. Our principal component analysis showed

483 that all four fungal treatments clustered together and away from the control (Supplementary Fig.

484 S7). However, there was no observed clustering by isogenic strain (NVP64 or NVP80) or by

485 cured/wild-type. Indeed, NVP64cu and NVP80wt seem to be the most similar.

486 DESeq2 provided p-values for each comparison, and we used the log2-fold change (LFC) and 487 adjusted $p$-value of the DESeq2 analyses to filter the expression patterns in the final DEG list. 488 DESeq2 identified a total of 465 genes that were differentially expressed and met LFC and 489 adjusted p-value cutoffs in at least one of the four fungal treatments as compared to the control. 490 Of these, there were 301 differentially expressed genes (DEGs) in NVP64cu v. Control, 135 in 491 NVP64wt v. Control, 142 in NVP80cu v. Control, and 213 in NVP80wt v. Control (Supplementary 492 Fig. S8). EdgeR identified 679 genes as being differentially expressed at a collective adjusted p493 value threshold, with at least one sample meeting the LFC cutoff. There were 376 DEGs in 494 NVP64cu v. Control, 240 in NVP64wt v. Control, 282 in NVP80cu v. Control, and 319 in NVP80wt 495 v. Control. We identified 385 DEGs present in both the EdgeR and DESeq2 differentially 496 expressed genes results (Supplementary Table S8; Fig. 6).

497 Thirty-four plant genes were differentially expressed when inoculated with all of the four fungal 498 treatments as compared to the uninoculated control, 55 in three fungal treatments, 114 in at least 499 two fungal treatments, and 182 in only one fungal treatment (Supplementary Table S8). 500 Differentially expressed genes responded in the same direction to treatments, with only one 501 exception (Supplementary Table S8). Table 2 highlights a subset of twenty five DEGs having 502 particularly interesting gene function and consistent significance across multiple fungal 503 treatments.

\section{Gene Ontology enrichment of differentially expressed genes}

505 Next, we ran Gene Ontology enrichment analysis on the differentially expressed genes (DEGs)

506 that responded to at least a single fungal inoculation. There were 172 upregulated and 212

507 downregulated genes. Several biological processes were enriched among these DEGs (Fig. 7).

508 In response to fungal treatment, upregulated genes were strongly enriched for "response to

509 oxidative stress", "defense response to bacterium", and notably "defense response to fungus".

510 Down regulated genes were enriched for "response to extracellular stimulus" and "response to

511 toxic substance". Broadly, these functional enrichments suggest external stimuli pathways were

512 highly fluctuating in response to fungal treatment. 


\section{Discussion}

514 In this study, root symbiosis between Arabidopsis thaliana and Linnemannia elongata were 515 characterized at the gene expression level and plants were phenotype for aerial plant growth and 516 seed production. We were also able to compare the impact of strain and endosymbiont (BRE vs.

517 MRE) on plant-fungal interactions since the two different $L$. elongata strains used harbored a 518 different endosymbiont. Finally, we used RNA-seq to identify plant genes that are differentially 519 expressed during Arabidopsis-L. elongata symbiosis in order to begin describing molecular 520 mechanisms of interaction associated with plant growth promotion.

\section{Linnemannia elongata promotes Arabidopsis growth independent of endobacteria}

522 This is the first study to explicitly test the impact of endobacteria on Linnemannia-plant 523 associations. We found that $L$. elongata increased aerial plant growth compared to uninoculated 524 controls, irrespective of the presence of endobacterial and independent of harvesting before or 525 after flowering. These growth promotion effects of Linnemannia are corroborated by recent studies of L. elongata inoculated maize, where L. elongata increased the height and dry aerial biomass of maize in V3-V5 early vegetative stages, which corresponds to when maize has begun relying on photosynthesis and the environment for resources, rather than seed resources $[1,46]$. Both MRE and BRE infection negatively impacts the growth of Linnemannia, thus, it is interesting that neither BRE nor MRE had a significant impact on plant growth in either experimental system $[16,17]$. However, NVP80wt (with MRE) did show a weak trend towards smaller plants than NVP80cu in the potting mix experiment (Fig. 2).

533 Previous studies have shown the L. elongata increases Calibrachoa flower production [45]. We 534 demonstrate here that $L$. elongata fungal treatments had a strong positive effect on Arabidopsis seed size and total seed number. This may be an important plant trait to consider when assessing 536 fitness costs of plant-associated microbes. Both NVP80cu and NVP80wt treatments had 537 significantly higher total seed number compared to uninoculated millet controls, however, this was 538 not the case for NVP64 inoculated treatments indicating strain variation. Interestingly, 539 uninoculated millet control plants had a higher average seed size compared to some of the fungal 540 treatments (Fig. 3b). While it is difficult to draw strong conclusions with so few replicates, it would 541 be interesting to specifically test whether this represents a fitness strategy in which plants grown 542 under stressful conditions create fewer, larger seeds to increase offspring fitness, whereas 543 healthy plants can produce a higher number of smaller seeds because each will need fewer 544 starting resources to survive and reproduce $[47,48]$. 


\section{Linnemannia elongata colonizes Arabidopsis root cells}

546 Following extended co-culture of Arabidopsis and L. elongata, hyphae of L. elongata were 547 observed to colonize root cells of Arabidopsis intracellularly. The intracellular hyphae were 548 contained within epidermal cells, including those both with and without root hairs (Fig 5). Stained 549 roots appeared healthy, but we are unable to determine if the root cells containing hyphae were 550 still alive, or any distinctive function of intracellular hyphae. Intracellular hyphae are especially 551 known from arbuscular mycorrhizal fungi, which produce highly branched arbuscules within the 552 root cells of their host and provide an extensive exchange surface for nutrients [49]. As opposed 553 to hyphae contained solely to the apoplast, these intracellular hyphae suggest a stronger 554 relationship between the host and fungus which could allow for exchange of nutrients, 555 phytohormones, or other metabolites. Yet, Serendipita (Piriformospora) indica requires dead root 556 cells for entrance into roots, but still provides benefits to the host [50]. Without further experiments 557 to characterize nutrient exchange through these intracellular structures, it is not possible to 558 ascribe structure with function [51].

559 Linnemannia elongata may regulate Arabidopsis defense and abiotic stress 560 responses

561 Up-regulated gene enrichments allow us to speculate on the transcriptional response of $A$. 562 thaliana to Linnemannia. Several genes in the peroxidase superfamily III were upregulated in 563 response to fungal inoculation. Nearly all these genes contain a signal peptide tagging them for 564 export out of the cell [52]. Indeed, some are known to be involved in cell wall remodeling [53,54], 565 a process that must occur to establish mutualism. However, they are also involved in defense 566 responses against pathogens. We propose a few alternative hypotheses why previously 567 described defense responses are upregulated in a mutualistic interaction. First, the assigned gene 568 ontology may inaccurately reflect the true function of these genes. They may be involved in 569 mutualism, but if it was not previously shown, these genes will lack that GO term. Second, the 570 upregulation of genes involved in defense response might be a priming response by $A$. thaliana 571 as previously shown by Johnson et al. [12].

572 A number of plant hormones mediate the initiation and maintenance of plant-microbe symbioses, 573 including auxins (most commonly IAA), jasmonates/jasmonic acid (JA), salicylic acid (SA), 574 abscisic acid (ABA), ethylene (ET), and brassinosteroids (BRs). These hormones can be 575 produced by both the plant and microbial symbionts and are often required to appropriately 576 suppress and redirect the plant defense response in order for the microbe to establish symbiosis. 
577 The regulation and importance of each hormone is specific to the type of interaction (e.g.

578 pathogen vs. mutualists) as well as the species of plant and microbe that are interacting. For

579 example, ethylene suppresses AMF colonization, but promotes EM colonization [55-57].

580 Similarly, the beneficial non-mycorrhizal fungi L. hyalina and Serendipita (=Piriformospora) indica

581 stimulate plant production of jasmonic acid and salicylic acid, respectively, when initiating

582 symbiosis with Arabidopsis [58,59]. While this study did not include direct measurement of

583 phytohormone levels, we did identify several DEGs related to the biosynthesis and signaling of

584 ethylene, auxin, and abscisic acid, which are discussed below.

\section{$585 \quad$ Root Development and Auxin}

586 We observed many upregulated genes in response to fungal inoculation that were previously shown to be upregulated during root development. This is interesting since development and stress response pathways overlap in plants [60,61]. Many fungi synthesize and secrete auxin, a hormone well known to impact plant growth. Podila verticillata (=Mortierella verticillata) and $M$. antarctica both synthesize IAA and were shown to improve winter wheat seedling growth [15]. The genome of $L$. elongata (strain AG77) has the key genes of IAA synthesis and maize roots inoculated with $L$. elongata had a $37 \%$ increase in IAA concentration [1]. Our study found that $L$. elongata suppressed Arabidopsis auxin biosynthesis genes (NIT2 and GH3.7), but up-regulated several auxin-responsive genes. Given that 1) Arabidopsis auxin biosynthesis is being downregulated, 2) auxin synthesis is generally self-inhibitory in plants, and 3) auxin response genes are up-regulated, we hypothesize that the Arabidopsis roots are responding to L. elongata-derived auxins [62]. However, Arabidopsis auxin-related genes did not respond to initial or established $L$. hyalina colonization, even though Arabidopsis roots had a 3-fold increase in IAA concentration during initial colonization as compared to control roots [12,58]. Moreover, some IAA was of fungal origin, as $L$. hyalina mycelium alone had a significantly higher IAA concentration than the tested pathogenic fungi [58]. It is worth noting that their assay did show a very brief response to auxin

602 that quickly dissipated to background gene expression levels. Since we found increased auxin 603 responsive gene expression during well-established symbiosis, our data indicate L. elongata may 604 employ a different phytohormone regulatory strategy compared to L. hyalina.

605 Enhanced aerial plant growth by auxin-producing microbes is attributed to improved root 606 structure, particularly lateral root growth, but assessing the impact of Mortierellaceae fungi on 607 plant root development is not so straightforward [1]. Johnson et al. [12] found that $L$. hyalina had 608 a slight, but significant negative impact on Arabidopsis root dry biomass compared to 609 uninoculated plants; they identified three root development (SHR, CPC, and AHP6) genes that 
610 responded to L. hyalina as opposed to the plant pathogen Alternaria brassicae. These genes

611 were not among the DEGs identified in our study. However, we did find that the entire operon-like

612 gene set related to thalianol biosynthesis and metabolism (MRN1, MRO, THAS1, THAH, and

613 THAD) was downregulated by L. elongata [63-66]. Thalianol-related metabolites are predicted to

614 function in promoting root development, but the mechanism is still under investigation [63]. Future

615 research is needed to determine how each of these fungi impact Arabidopsis root development

616 and how that relates to increased aerial plant growth.

\section{Ethylene (ET)}

618 Ethylene is a plant hormone involved in maturation, senescence, and response to biotic and 619 abiotic stress. Decreasing the level of ethylene in plant tissues generally promotes plant growth.

620 The role of ET in plant response to pathogens is well characterized and includes increased ET

621 biosynthesis and signaling through a single conserved pathway, which includes proteins in the 622 TDR1 family [67]. However, the origin and role of ET in the initiation of beneficial plant-fungal 623 symbioses is specific to the fungi involved. For instance, elevated ET appears to promote 624 colonization by ectomycorrhizal fungi, but inhibits colonization by AMF [55-57]. Moreover, the ET 625 signaling pathway is known to have multiple points of crossover with other hormone signaling 626 pathways, including JA and cytokinin, some of which occur through the ERF family of transcription 627 factors, including TDR1 [67]. In our study, we found that Arabidopsis colonized by L. elongata 628 down-regulated ACC synthase ACS7, which synthesizes the metabolite 1-amino-cyclopropane629 1-carboxylate (ACC), a precursor of ethylene. However, some genes related to ethylene signaling 630 were up-regulated in response to L. elongata. Since ET biosynthesis is downregulated in 631 Arabidopsis roots in response to L. elongata, it is possible that related response genes are up632 regulated via other hormone pathways. There were only three DEGs specifically associated with 633 JA signaling in our dataset but they were each significant in only one fungal treatment.

\section{Abscisic acid (ABA), abiotic stress, \& reactive oxygen species (ROS)}

635 In general, we found that genes related to ABA and abiotic stress are down-regulated by $L$. 636 elongata. These include the ABA synthesis enzyme NCED3 and responses to drought, cold, salt, 637 iron deficiency, potassium deficiency, phosphorous deficiency, and heavy metals. Many plant 638 growth promoting fungi are thought to transport water and nutrients to plants, particularly 639 phosphorus. Mortierellaceae species are known to solubilize phosphate and improve phosphorus 640 uptake in plants [68]. Considering the availability of potassium, phosphorus, and iron in the PNM 641 growth medium, it is striking that so many genes related to deficiencies of these nutrients were 642 down-regulated compared to the control plants. There were two main exceptions to this reduction 
643 in abiotic stress: oxidative stress responses and a group of RmIC-like cupins superfamily proteins

644 whose function is unknown.

645 ROS are a common plant defense response to both beneficial and pathogenic microbes [69]. Both

646 L. hyalina and L. elongata stimulate ROS-responsive genes, although the two ROS-responsive

647 genes specifically tested by Johnson et al. [12] were not among the DEGs in our dataset. Six of

648 the up-regulated oxidative stress genes were peroxidases. One was a raffinose synthase.

649 Raffinose is thought to act as an osmoprotectant and ROS scavenger [70]. Finally, we observed

650 down-regulation of uridine diphosphate glycosyltransferase UGT74E2, which responds to ROS

651 and drought to convert the auxin IBA to IBA-GIc (Tognetti et al. 2010). ROS also stimulates

652 conversion of IAA to IBA. Increased expression of UGT74E2 further sequesters IBA and prevents

653 oxidation back to IAA [71]. While no UGT74E2 suppression or deletion mutant phenotypes have

654 been reported, overexpression of UGT74E2 leads to increased sensitivity to ABA [72]. In

655 summary, we observe down-regulation of auxin synthesis, ABA synthesis and signaling, and an

656 important gene connecting the ROS, ABA, and auxin pathways. From this, we infer that $L$.

657 elongata stimulates ROS responsives genes, but these responses are isolated from other

658 hormone pathways and limited to peroxidases and antioxidants.

\section{Calcium signaling and plant defense}

660 In addition to hormones, many plant-microbe interactions involve calcium signaling in plant roots

661 [73]. L. hyalina symbiosis with Arabidopsis is activated by calcium signaling [12]. Calcium-

662 signaling was required for the plants to receive pathogen protection by $L$. hyalina, and for $L$.

663 hyalina to colonize Arabidopsis roots; however, signaling-deficient plants still showed the wild-

664 type aerial growth promotion. This suggests a calcium-signaling dependent defense response to

665 limit the rate of root colonization by L. hyalina. Johnson et al. [12] identified four Ca-signaling

666 genes (At3g47480, At3g03410, At5g23950, and At3g60950) that specifically responded to $L$.

667 hyalina, as opposed to the plant pathogen Alternaria brassicae. These genes were not among

668 the DEGs identified in our study. However, our RNA-seq experiment did demonstrate an up-

669 regulation of the calcium-signaling gene CML12, which is induced by both stress and hormone

670 signals, including auxin, touch, darkness, oxidative stress, and herbivory [74,75].

671 DEG analyses indicate that $L$. elongata stimulated several general, fungal, and bacterial defense-

672 related genes in Arabidopsis roots. However, we also noted suppression of genes involved in

673 programmed cell death and production of defensin-like proteins meant to kill cells of invading

674 organisms. As such, these defense responses could indicate both regulation of L. elongata

675 colonization and a priming of the plant innate immune response, explaining the elevated 
676 expression of definitively bacterial defense genes like FLS2. As noted in maize-L. elongata

677 symbioses, L. elongata may curate auxin levels to colonize maize roots and suppress systemic

678 defense through the salicylic acid pathway [1]. Further, this active microbial regulation of the plant

679 immune response may promote plant growth in a field environment by limiting further resource

680 allocation to defense when attacked by pathogens $[1,76]$.

\section{Challenges of plant-fungi experiments}

682 There are challenges to introducing fungi to plants without simultaneously altering other factors.

683 Experiments carried out in potting mixes reiterate that uninoculated grains in control treatments 684 not only invite colonization by environmental contaminants, but the grains themselves may 685 introduce a strong and consistent negative impact on plant growth. However, we found the potting 686 mix experiment was technically sufficient to collect data about seed production, while the agar inoculation approach allowed for more controlled growth. Now that $L$. elongata has been verified to impact plant growth under these conditions, more extensive experiments can be justified to throughput assays of plant and fungal knock-out mutants to further isolate important genes and pathways involved in this symbiosis, and for assaying early life-stage aerial growth and root gene expression. However, semi-solid rooting conditions may not be representative of plants grown in soil or more real-world conditions. An improved potting mix system based upon a grain-free inoculation protocol would be ideal to non-destructively track plant growth over time and to construct a more detailed description of how L. elongata affects plant growth and development.

\section{The role of phytohormones in fungi}

697 While it is well established that fungi can manipulate and produce phytohormones, the effects of 698 phytohormones on fungi are not well understood. Studies of plant hormone impacts on fungal 699 growth and development are currently limited to a few plant pathogens and AM fungi. Exogenous 700 ethylene is known to promote fungal spore germination and mycelial growth [77-79]. For example, 701 exogenous ethylene is required for spore germination in species of Alternaria, Botrytis, 702 Penicillium, and Rhizopus and often promotes mycelial growth [79]. It is worth noting that these 703 fungal species infect fruit, and likely evolved through selection for spore germination in the 704 presence of ripening fruit, limiting the relevance of those findings to mechanisms employed by 705 root-associated fungi [78]. Gryndler et al. [80] found that exogenous auxin (IAA) repressed hyphal 706 growth of two AM fungi, Glomus fistulosum and G. mosseae, at biologically relevant 707 concentrations, but abscisic acid (ABA) and cytokinins had no perceivable effect until applied in 708 concentrations very high, non-physiologically relevant, concentrations. The current model of 
709 phytohormone regulation of AM fungi suggests that 1) SA inhibits pre-symbiotic growth; 2)

710 ethylene, JA, and cytokinins inhibit symbiotic fungal growth inside plant roots; and 3) auxin, JA,

711 and ABA promote the formation and function of arbuscules within plant root cells [81]. It is still

712 unclear how these relationships and regulatory systems apply to the growth, development, or

713 plant associations of $L$. elongata, but these are important questions to consider in future plant-

714 fungal interaction studies.

\section{Conclusions}

716 In conclusion, we phenotyped Arabidopsis at early and late life stages during a stable symbiosis 717 with Linnemannia in soil and agar-based media. We demonstrated that $L$. elongata promotes 718 Arabidopsis above-ground vegitative growth and seed production. This plant phenotype was 719 found to be independent of whether $L$. elongata isolates were colonized by MRE or BRE 720 endohyphal bacterial symbionts. We hypothesize that the mechanism of plant-fungal symbiosis 721 involves fungal production of auxin and stimulation of the ethylene and ROS response pathways. 722 Future research is needed to test these hypotheses and further characterize the fungal side of 723 this symbiosis.

\section{Acknowledgements}

725 We would like to thank Abigail Bryson and Bryan Rennick for their extensive assistance with setting up experiments, Xinxin Wang for assistance collecting Arabidopsis seeds from plant material, and Natalie Golematis for help with antibiotic passaging to cure fungal strains and DNA extractions for qPCR analyses. We would like to thank Dr. Zsofia Szendrei for generously providing access to her lab microbalance for weighing seeds and plants. We are grateful to Keith Koonter and Dr. Matthew Greishop for sharing their automated image analysis pipeline. Funding sources: GB acknowledges support from the US National Science Foundation DEB 1737898 and the US Department of Agriculture NIFA MICL02416.

\section{Author Contributions}

735 NVP - experiment design \& completion, data collection, data analysis, manuscript writing

$736 \mathrm{JL}$ - generation of cured fungal isolates, qPCR, root harvesting, root microscopy, manuscript 737 writing

738 AY - gene ontology analysis, manuscript writing 
JM - statistical analysis of plant biomass data

PE - supported RNASeq exp design \& data analyses, manuscript writing

GB - research support, experiment design, manuscript writing

\section{Figure \& Table Captions}

744 Figure 1 - Arabidopsis plants at the time of harvest for aerial biomass assay

745 Ten days after germination, Arabidopsis thaliana seedlings were transplanted from 1xMS 746 germination plates to these PNM plates and inoculated with small blocks of Kaefer Medium, either 747 colonized by fungi (left) or sterile (right). The Arabidopsis (and fungi, when applicable) grew on 748 PMN plates for 12 days, at which point these pictures were captured and the plants harvested for 749 aerial biomass assays.

Arabidopsis thaliana was grown to maturity and the aerial biomass harvested and dried. Treatments refer to the composition of the potting mix. The untreated control (NoMillet) contrasted

754 treatments where the sterile potting soil was mixed 97:3 v:v with sterile millet mix (Uninoculated), 755 or millet mix inoculated with one of four Linnemannia elongata strains (NVP64cu, NVP64wt, 756 NVP80cu, or NVP80wt). Colors correspond to treatment, horizontal brackets and numbers 757 indicate pairwise Wilcox ranked sum tests and the resulting p-value. $\mathrm{N}=12$ for all treatments. 758 Between NVP64cu v. NVP64wt, NVP80cu v. NVP80wt, and NoMillet v. Uninoculated, we used 759 two-tailed tests. Between each fungal treatment and the Uninoculated, we performed one-tailed 760 tests with the alternative hypothesis "greater".

Figure 3 - Total and Average Arabidopsis seed mass collected in potting mix experiments

763 Arabidopsis thaliana was grown to maturity and the seeds collected by Aracon tubes. Treatments

764 refer to the composition of the substrate in which Arabidopsis plants were grown. The NoMillet 765 Control was autoclaved SureMix. All other treatments were autoclaved SureMix substrate mixed 766 97:3 v:v with sterile grain-based inoculum (Uninoculated), or grain-based inoculum colonized by 767 one of four Linnemannia elongata strains (NVP64cu, NVP64wt, NVP80cu, or NVP80wt). N=5 for 768 all treatments. Colors correspond to treatment, horizontal brackets and numbers indicate pairwise 
Wilcox ranked sum tests and the resulting p-value. Between NVP64cu v. NVP64wt, NVP80cu v. NVP80wt, and NoMillet v. Uninoculated, we used two-tailed tests. Between each fungal treatment and the Uninoculated, we performed one-tailed tests with the alternative hypothesis "greater" for total seed mass \& "less" for average seed mass. a) Total seed mass collected. b) Average seed mass was determined by weighing and then counting a subset of seeds taken from the total seed mass.

Figure 4 - Linnemannia elongata colonization of Arabidopsis increased aerial dry weight in agar-

777 based interaction experiments

778 The estimated marginal mean of Arabidopsis thaliana aerial dry weight, modeled as a function of 779 starting root length and treatment with nested effects for experiment iteration (3 independent 780 iterations) and agar plate (3 plants per plate). Treatments included the uninoculated control and 781 four strains of Linnemannia elongata. $\mathrm{N}=39$ plates for Control, NVP64wt, and NVP80wt, $\mathrm{nN}=69$ 782 plates for NVP64cu and NVP80cu. The degrees of freedom for each comparison were 783 approximated using the kenward-roger method and the p-values adjusted for multiple comparisons using the Tukey method for comparing a family of 5 estimates. Letters indicate significantly different groups with an alpha value of 0.05 . Exact values can be found in Table 1 .

Figure 5 - Colonization of Arabidopsis roots by L. elongata.

a-f) NVP64cu at 25 days post inoculation, $\mathbf{g}-\mathbf{I}$ ) NVP80cu at 23 days post inoculation. a,g) Fluorescence signal from calcofluor white M2R staining; $\mathbf{b}, \mathbf{h}$ ) signal from wheat germ agglutinin 640R staining c-f,i-I) merged fluorescence. d-f,j-I) Orthogonal z-stack projections of root micrographs. a-I) White arrows indicate plant wall structures showing hyphae (blue) contained within intracellular spaces by plant cell walls (red). Scale bars represent $20 \mu \mathrm{m}$.

Figure 6 - Abundance of differentially expressed Arabidopsis genes threshold of 1.5 and p-value threshold of 0.05. a) A Venn diagram of all DEGs in the final, filtered 
Figure 7 - GO enrichment of up and down regulated genes

802 The ten GO categories with the strongest enrichment are displayed for both a) up and b) down

803 regulated genes in response to fungal treatment. Color corresponds to the adjusted $p$-value 804 according to the Benjamini-Hochberg Procedure while dot size corresponds to the number of 805 differentially expressed genes matching a given GO category.

807 Supplementary Figure S1-Arabidopsis seedlings used in plant-fungal interaction assays

808 Panel a) 10-day old Arabidopsis thaliana seedlings on 1xMS germination plates and b) 11-day 809 old Arabidopsis seedlings and blocks of media (colonized by fungi in fungal treatments or sterile 810 in uninoculated control treatments) as arranged on PNM plates for the agar-based plant-fungal 811 interaction experiments.

813 Supplementary Figure S2 - Seeds to be counted by image analysis

814 Arabidopsis thaliana was grown to maturity and the seeds of each plant collected by Aracon tubes

815 and stored in Eppendorf tubes. After careful cleaning of the seed to remove stems, petals, and 816 other plant debris, approximately $14 \mathrm{mg}$ of seeds per sample were weighed on an ultrasensitive 817 balance, adhered to a piece of white paper using a glue stick, covered by clear packing tape, 818 scanned, and counted by image analysis in ImageJ. a) The scanned image of the subsampled 819 seeds. b) The image analysis output, with areas identified as a "seed" outlined in red.

821 Supplementary Figure S3 - Agar plates with Arabidopsis plants in the growth chamber

822 Arabidopsis thaliana seeds germination and Arabidopsis-L. elongata interaction studies were 823 conducted on agar plates. These were incubated in a Percival growth chamber. Plates were 824 stacked on a gentle angle to encourage smooth directional root growth along the agar surface.

826 Supplementary Figure S4 - Bolting phenotype

827 The arrow indicates the elongation of the Arabidopsis thaliana inflorescence away from the rosette 828 of leaves which was considered to indicate "bolting." 


\section{Supplementary Figure S5 - Violin plots of Arabidopsis seed image area}

831 Arabidopsis thaliana was grown to maturity and the seeds collected by Aracon tubes. Facet

832 names indicate the composition of the potting mix in which Arabidopsis plants were grown. The

833 untreated control (NoMillet) contrasted treatments where the sterile potting soil was mixed 97:3

834 v:v with sterile millet mix (Uninoculated), or millet mix inoculated with one of four Linnemannia

835 elongata strains (NVP64cu, NVP64wt, NVP80cu, or NVP80wt). A subset of seeds from 5 samples

836 per treatment (sample indicated by 'Rep') were adhered to white paper and imaged using an

837 Epson scanner. The $y$-axis indicates the pixel count of each individual seed scanned for each rep

838 and treatment using ImageJ.

840 Supplementary Figure S6 - Linnemannia elongata strains equivalently colonized Arabidopsis

841 roots

842 RNA was extracted from Arabidopsis thaliana roots colonized by Linnemannia elongata, pooled

843 from all three plants on each agar plate, from three plates per treatment. The inferred ratio of

844 fungal:plant cDNA is based on the qPCR results and standard curves for each qPCR primer set.

845 Since Arabidopsis GADPH and L. elongata RPB1 are single copy genes, the ratio of fungal and

846 plant template provides a normalized estimate of fungal colonization of plant roots.

848 Supplementary Figure S7 - Principal component analysis of differential Arabidopsis gene

849 expression

850 Arabidopsis thaliana root RNAseq data analyzed using DESeq2, sequenced from three biological 851 replicates taken from each of the uninoculated control and fungal treatments inoculated with 852 Linnemannia elongata.

854 Supplementary Figure S8-Volcano plots of differential gene expression

855 Pairwise comparisons of normalized Arabidopsis thaliana gene expression between fungal 856 treatments and the uninoculated control, calculated from the DESeq2 analyses. Each point 857 represents a gene, plotted by adjusted p-value and Log2 Fold Change (LFC) in expression 858 between the fungal treatment and the control. Vertical dashed lines indicate the $|\mathrm{LFC}|=1$ threshold 859 and horizontal lines indicate the adjusted p-value threshold of 0.05 used to identify genes with 
significant changes in expression. Genes are colored by which of the LFC and p-value cutoffs were exceeded: gray = failed both; green = exceeded only the LFC cutoff, but not the p-value cutoff; blue = exceeded p-value cutoff, but not LFC; red = exceeded both cutoffs.

Table 1 - Linear mixed modeling of Arabidopsis aerial dry weight

To account for having measurements for three plants per agar plate and two independent repetitions of the agar-based interaction experiment, experimental round and plate were treated as random/grouping effects. The starting root length and experimental treatment were fixed effects, where the uninoculated control treatment was estimated as the intercept.

872 Table 2 - Subset of Arabidopsis genes differentially expressed in response to Linnemannia 873 elongata

874 A subset of twenty five DEGs having particularly interesting gene function and consistent 875 significance across multiple fungal treatments. Log 2 fold change (LFC) values were calculated 876 by DESeq2 and filtered at $|\mathrm{LFC}|=\log 2(1.5)=0.58$ and adjusted $p$-value $=0.05$. Table is organized 877 first by functional annotation, then by direction of regulation, and finally by the number of fungal 878 treatments in which the gene was differentially expressed.

Supplementary Table S1 - A map of the light levels in the growth chamber

881 Arabidopsis thaliana seeds germination and Arabidopsis-L. elongata interaction studies were 882 conducted on agar plates. These were incubated in a Percival growth chamber. Each shelf in the 883 chamber was divided into nine regions and the light level in each region was measured using an 884 LI-250A light meter (LI-COR) with the chamber door closed to ensure realistic conditions. Light 885 levels on the middle and bottom shelves were measured after arranging agar plates on the above 886 shelf/shelves.

889 The qPCR primer sets used to quantify fungal colonization of plant roots and check for BRE/MRE 
890 in cured and wild-type fungal strains and fungus-colonized plant roots.

892 Supplementary Table S3 - Linear modeling of Arabidopsis aerial dry weight as a function of light

893 level

894 The aerial dry biomass of Arabidopsis thaliana plants harvested from agar-based Arabidopsis-

895 Linnemannia elongata interaction experiments and modeled as a function of light level. Medium

896 indicates the composition of the medium on which L. elongata was cultured: KM = Kaefer Medium;

897 MEA = Malt Extract Agar. Treatment indicates the strain of $L$. elongata with which Arabidopsis

898 was inoculated or the uninoculated control.

900 Supplementary Table S4 - Linear modeling of Arabidopsis aerial dry weight as a function of 901 treatment and medium

902 The aerial dry biomass of Arabidopsis thaliana plants from agar-based Arabidopsis-Linnemannia 903 elongata interaction experiments, modeled as a function of treatment (control v. different strains 904 of Linnemannia elongata), the medium on which the fungi had been cultured, and any interaction 905 between those terms. We also conducted pairwise comparisons within treatments of the 906 estimated marginal means (EMMs) for each inoculating medium.

908 Supplementary Table S5 - Linear modeling of Arabidopsis aerial dry weight as a function of 909 starting seedling root length

910 The aerial dry biomass of Arabidopsis thaliana plants from agar-based Arabidopsis-Linnemannia 911 elongata interaction experiments, modeled as a function of seedling starting root length. There 912 were no significant differences in the slope of the relationship of starting root length to final aerial 913 dry weight across experimental rounds or treatments.

915 Supplementary Table S6 - qPCR of plant, fungal, and endobacterial genes from RNA

916 Values indicate the mean $(n=2)$ qPCR cycle number at which amplification reached the threshold 917 of detection $\left(C_{t}\right)$ for each locus tested in each cDNA library from the Arabidopsis thaliana root 918 RNA samples used in the RNAseq experiment. Dash = not tested. Arabidopsis GADPH and 919 Linnemannia elongata RPB1 are single copy genes. The bacterial 16S gene is multicopy, which 
920 was necessary for detection, since these endobacteria are in very low abundance in the fungal

921 hyphae.

922

923 Supplementary Table S7 - Molecular results of RNA sequencing run

924 The number of sequenced reads passing initial filtration by the sequencer, the percentage of

925 those reads that mapped to the combined reference transcriptome, and the proportion of mapped

926 reads that mapped to plant or fungal transcripts.

928 Supplementary Table S8 - Arabidopsis genes differentially expressed in response to

929 Linnemannia elongata

930 Log 2 fold change (LFC) values were calculated by DESeq2 and filtered at $\mid$ LFC $\mid=\log 2(1.5)=0.58$ 931 and adjusted $p$-value $=0.05$. Table is organized first by functional annotation, then by direction of

932 regulation, and finally by the number of fungal treatments in which the gene was differentially 933 expressed.

\section{$936 \quad$ Literature Cited}

937 1. Li F, Chen L, Redmile-Gordon M, Zhang J, Zhang C, Ning Q, et al. Mortierella elongata 's 938 roles in organic agriculture and crop growth promotion in a mineral soil. Land Degrad Dev. 2018;29: 1642-1651.

940 2. Bedini A, Mercy L, Schneider C, Franken P, Lucic-Mercy E. Unraveling the Initial Plant 941 Hormone Signaling, Metabolic Mechanisms and Plant Defense Triggering the 942 Endomycorrhizal Symbiosis Behavior. Front Plant Sci. 2018;9: 1800.

943 3. Spatafora JW, Chang Y, Benny GL, Lazarus K, Smith ME, Berbee ML, et al. Zygomycete 944 Genealogy of Life (ZyGoLife): a phylum-level phylogenetic classification of zygomycete 945 fungi based on genome-scale data. Mycologia. 2016.

946 4. Hooker JE, Jaizme-Vega M, Atkinson D. Biocontrol of plant pathogens using arbuscular 947 mycorrhizal fungi. In: Gianinazzi S, Schüepp H, editors. Impact of Arbuscular Mycorrhizas 
on Sustainable Agriculture and Natural Ecosystems. Basel: Birkhäuser Basel; 1994. pp. 191-200.

5. Tedersoo L, May TW, Smith ME. Ectomycorrhizal lifestyle in fungi: global diversity, distribution, and evolution of phylogenetic lineages. Mycorrhiza. 2010;20: 217-263.

6. Vandepol N, Liber J, Desirò A, Na H, Kennedy M, Barry K, et al. Resolving the Mortierellaceae phylogeny through synthesis of multi-gene phylogenetics and phylogenomics. Fungal Divers. 2020;104: 267-289.

7. James TY, Kauff F, Schoch CL, Matheny PB, Hofstetter V, Cox CJ, et al. Reconstructing the early evolution of Fungi using a six-gene phylogeny. Nature. 2006;443: 818-822.

8. Chang Y, Desirò A, Na H, Sandor L, Lipzen A, Clum A, et al. Phylogenomics of Endogonaceae and evolution of mycorrhizas within Mucoromycota. New Phytol. 2019;222: 511-525.

9. Stiles W. On the Relation between the Concentration of the Nutrient Solution and the Rate of Growth of Plants in Water Culture. Ann Bot. 1915;29: 89-96.

10. Bisby GR, Timonin MI, James N. Fungi isolated from soil profiles in Manitoba. Can J Res. 1935;13c: 47-65.

11. Domsch KH, Gams W, Anderson T-H, Others. Compendium of soil fungi. Volume 1. Academic Press (London) Ltd.; 1980.

12. Johnson JM, Ludwig A, Furch ACU, Mithöfer A, Scholz S, Reichelt M, et al. The Beneficial Root-Colonizing Fungus Mortierella hyalina Promotes the Aerial Growth of Arabidopsis and Activates Calcium-Dependent Responses That Restrict Alternaria brassicae--Induced Increases Plant Biomass among Non-Leguminous Crop Species. Agronomy. 2020;10: 754. endophytes of Populus trichocarpa alter host phenotype, gene expression and rhizobiome composition. Molecular Plant-Microbe Interactions. 2019. doi:10.1094/mpmi-05-18-0133-r

975 15. Ozimek E, Jaroszuk-Ściseł J, Bohacz J, Korniłłowicz-Kowalska T, Tyśkiewicz R, Słomka A, 
et al. Synthesis of Indoleacetic Acid, Gibberellic Acid and ACC-Deaminase by Mortierella Strains Promote Winter Wheat Seedlings Growth under Different Conditions. Int J Mol Sci. 2018;19. doi:10.3390/ijms19103218

16. Desirò A, Hao Z, Liber JA, Benucci GMN, Lowry D, Roberson R, et al. Mycoplasma-related endobacteria within Mortierellomycotina fungi: diversity, distribution and functional insights into their lifestyle. ISME J. 2018;12: 1743-1757.

17. Uehling J, Gryganskyi A, Hameed K, Tschaplinski T, Misztal PK, Wu S, et al. Comparative genomics of Mortierella elongata and its bacterial endosymbiont Mycoavidus cysteinexigens. Environ Microbiol. 2017;19: 2964-2983.

18. Ohshima S, Sato Y, Fujimura R, Takashima Y, Hamada M, Nishizawa T, et al. Mycoavidus cysteinexigens gen. nov., sp. nov., an endohyphal bacterium isolated from a soil isolate of the fungus Mortierella elongata. Int J Syst Evol Microbiol. 2016;66: 2052-2057.

19. Okrasinska A, Bokus A, Duk K, Gęsiorska A, Sokołowska B, Miłobędzka A, et al. New Endohyphal Relationships between Mucoromycota and Burkholderiaceae Representatives. Appl Environ Microbiol. 2021;87. Available: https://www.researchgate.net/profile/Alicja-

20. Takashima Y, Seto K, Degawa Y, Guo Y, Nishizawa T, Ohta H, et al. Prevalence and IntraOkrasinska/publication/348762415_New_endosymbiotic_relationships_between_Mucorom ycota_and_Burkholderiaceae_representatives/links/604b60a6a6fdcc4d3e5a05bd/Newendosymbiotic-relationships-between-Mucoromycota-and-Burkholderiaceaerepresentatives.pdf

21. Salvioli A, Ghignone S, Novero M, Navazio L, Venice F, Bagnaresi P, et al. Symbiosis with an endobacterium increases the fitness of a mycorrhizal fungus, raising its bioenergetic potential. ISME J. 2016;10: 130-144.

22. Bonfante $P$, Desirò $A$. Who lives in a fungus? The diversity, origins and functions of fungal endobacteria living in Mucoromycota. ISME J. 2017. doi:10.1038/ismej.2017.21 
taxonomy".(Eds GM Hewitt, A Johnston) pp. 283--293. Springer: Berlin; 1991.

1006

1007

1008

1009

1010

1011

1012

1013

1014

1015

1016

1017

1018

1019

1020

1021

1022

1023

1024

1025

1026

1027

1028

1029

1030

1031

24. Ahlmann-Eltze C. Ggsignif: significance brackets for "ggplot2." R package version 040. 2019.

25. Kassambara A. ggcorrplot: Visualization of a correlation matrix using "ggplot2". R package version 0.1. 3. 2019.

26. R Development Core Team R. R: A Language and Environment for Statistical Computing. R Foundation for Statistical Computing; 2011. doi:10.1007/978-3-540-74686-7

27. Bates D, Mächler M, Bolker B, Walker S. Fitting Linear Mixed-Effects Models Usinglme4. Journal of Statistical Software. 2015. doi:10.18637/jss.v067.i01

28. Kuznetsova A, Brockhoff PB, Christensen RHB. ImerTest package: tests in linear mixed effects models. J Stat Softw. 2017;82: 1-26.

29. Fox J, Weisberg S, Price B, Adler D, Bates D, Baud-Bovy G, et al. car: Companion to Applied Regression. R package version 3.0-2. Website: https://cran r-proje ct org/web/packa ges/car/index html [accessed 01 June 2019]. 2019.

30. Wickham H, Averick M, Bryan J, Chang W, McGowan L, François R, et al. Welcome to the Tidyverse. Journal of Open Source Software. 2019. p. 1686. doi:10.21105/joss.01686

31. Kruskal WH, Wallis WA. Use of Ranks in One-Criterion Variance Analysis. J Am Stat Assoc. 1952;47: 583-621.

32. Lenth R. emmeans: Estimated marginal means, aka least-squares means (Version 1.5. 21)[R package]. 2020.

33. Schneider CA, Rasband WS, Eliceiri KW. NIH Image to ImageJ: 25 years of image analysis. Nat Methods. 2012;9: 671-675.

34. Fedorov A, Beichel R, Kalpathy-Cramer J, Finet J, Fillion-Robin J-C, Pujol S, et al. 3D Slicer as an image computing platform for the Quantitative Imaging Network. Magn Reson Imaging. 2012;30: 1323-1341.

35. Bolger AM, Lohse M, Usadel B. Trimmomatic: a flexible trimmer for Illumina sequence data. Bioinformatics. 2014;30: 2114-2120. 
36. Patro R, Duggal G, Love MI, Irizarry RA, Kingsford C. Salmon provides fast and bias-aware quantification of transcript expression. Nat Methods. 2017;14: 417-419.

37. Zhang R, Calixto CPG, Marquez Y, Venhuizen P, Tzioutziou NA, Guo W, et al. AtRTD2: A Reference Transcript Dataset for accurate quantification of alternative splicing and expression changes in Arabidopsis thaliana RNA-seq data. bioRxiv. 2016. p. 051938. doi:10.1101/051938

38. Robinson MD, McCarthy DJ, Smyth GK. edgeR: a Bioconductor package for differential expression analysis of digital gene expression data. Bioinformatics. 2010;26: 139-140.

39. McCarthy DJ, Chen Y, Smyth GK. Differential expression analysis of multifactor RNA-Seq experiments with respect to biological variation. Nucleic Acids Res. 2012;40: 4288-4297.

40. Love MI, Huber W, Anders S. Moderated estimation of fold change and dispersion for RNAseq data with DESeq2. Genome Biol. 2014;15: 550.

41. Blighe K, Rana S, Lewis M. EnhancedVolcano: Publication-ready volcano plots with enhanced colouring and labeling. R package version. 2019;1.

42. Berardini TZ, Reiser L, Li D, Mezheritsky Y, Muller R, Strait E, et al. The Arabidopsis information resource: Making and mining the "gold standard" annotated reference plant genome. Genesis. 2015;53: 474-485.

43. UniProt Consortium. UniProt: a worldwide hub of protein knowledge. Nucleic Acids Res. 2019;47: D506-D515.

44. Wu T, Hu E, Xu S, Chen M, Guo P, Dai Z, et al. clusterProfiler 4.0: A universal enrichment tool for interpreting omics data. Innovation (N Y). 2021;2: 100141.

45. Becker LE, Cubeta MA. Increased Flower Production of Calibrachoa $x$ hybrida by the Soil Fungus Mortierella elongata. J Environ Hortic. 2020;38: 114-119.

46. Abendroth LJ, Myers AJW, Elmore RW. Corn planting date: Understanding plant growth and yield response. Proceedings of the Integrated Crop Management Conference. 2009. doi:10.31274/icm-180809-1

47. Sadras VO. Evolutionary aspects of the trade-off between seed size and number in crops. Field Crops Res. 2007;100: 125-138. 
48. Breen AN, Richards JH. Irrigation and fertilization effects on seed number, size, germination and seedling growth: implications for desert shrub establishment. Oecologia. 2008;157: 13-19.

49. Genre A, Bonfante P. Building a mycorrhizal cell: How to reach compatibility between plants and arbuscular mycorrhizal fungi. Journal of Plant Interactions. 2005. pp. 3-13. doi:10.1080/17429140500318986

50. Deshmukh S, Hückelhoven R, Schäfer $P$, Imani J, Sharma $M$, Weiss $M$, et al. The root endophytic fungus Piriformospora indica requires host cell death for proliferation during mutualistic symbiosis with barley. Proc Natl Acad Sci U S A. 2006;103: 18450-18457.

51. Brundrett M. Diversity and classification of mycorrhizal associations. Biol Rev Camb Philos Soc. 2004;79: 473-495.

52. Valério L, De Meyer M, Penel C, Dunand C. Expression analysis of the Arabidopsis peroxidase multigenic family. Phytochemistry. 2004;65: 1331-1342.

53. Hiraga S, Sasaki K, Ito H, Ohashi Y, Matsui H. A large family of class III plant peroxidases. Plant Cell Physiol. 2001;42: 462-468.

54. Kwon T, Sparks JA, Nakashima J, Allen SN, Tang Y, Blancaflor EB. Transcriptional response of Arabidopsis seedlings during spaceflight reveals peroxidase and cell wall remodeling genes associated with root hair development. Am J Bot. 2015;102: 21-35.

55. Chanclud E, Morel J-B. Plant hormones: a fungal point of view. Mol Plant Pathol. 2016;17: 1289-1297.

56. Foo E, McAdam EL, Weller JL, Reid JB. Interactions between ethylene, gibberellins, and brassinosteroids in the development of rhizobial and mycorrhizal symbioses of pea. J Exp Bot. 2016;67: 2413-2424.

57. Splivallo R, Fischer U, Göbel C, Feussner I, Karlovsky P. Truffles regulate plant root morphogenesis via the production of auxin and ethylene. Plant Physiol. 2009;150: 20182029.

58. Meents AK, Furch ACU, Almeida-Trapp M, Özyürek S, Scholz SS, Kirbis A, et al. Beneficial and Pathogenic Arabidopsis Root-Interacting Fungi Differently Affect Auxin Levels and 
Responsive Genes During Early Infection. Frontiers in Microbiology. 2019. doi:10.3389/fmicb.2019.00380

59. Vahabi K, Sherameti I, Bakshi M, Mrozinska A, Ludwig A, Reichelt M, et al. The interaction of Arabidopsis with Piriformospora indica shifts from initial transient stress induced by fungus-released chemical mediators to a mutualistic interaction after physical contact of the two symbionts. BMC Plant Biol. 2015;15: 58.

60. Xia N, Zhang G, Liu X-Y, Deng L, Cai G-L, Zhang Y, et al. Characterization of a novel 1095 wheat NAC transcription factor gene involved in defense response against stripe rust pathogen infection and abiotic stresses. Mol Biol Rep. 2010;37: 3703-3712.

61. Qi J, Li J, Han X, Li R, Wu J, Yu H, et al. Jasmonic acid carboxyl methyltransferase regulates development and herbivory-induced defense response in rice. J Integr Plant Biol. 2016;58: 564-576.

62. Salehin M, Bagchi R, Estelle M. SCFTIR1/AFB-based auxin perception: mechanism and role in plant growth and development. Plant Cell. 2015;27: 9-19.

63. Field B, Osbourn AE. Metabolic Diversification-Independent Assembly of Operon-Like Gene Clusters in Different Plants. Science. 2008 [cited 10 Nov 2021]. doi:10.1126/science.1154990

64. Field B, Fiston-Lavier A-S, Kemen A, Geisler K, Quesneville H, Osbourn AE. Formation of plant metabolic gene clusters within dynamic chromosomal regions. Proc Natl Acad Sci U S A. 2011;108: 16116-16121.

65. Go YS, Lee SB, Kim HJ, Kim J, Park H-Y, Kim J-K, et al. Identification of marneral synthase, which is critical for growth and development in Arabidopsis. Plant J. 2012;72: 791-804.

66. Johnson EE. A profile of the expression of a metabolic gene cluster in Arabidopsis. University of British Columbia; 2012. doi:10.14288/1.0072908 pathogen interactions. Annu Rev Phytopathol. 2006;44: 393-416. 
phosphate-solubilizing fungus (Mortierella sp.) and their effects on Kostelelzkya virginica growth and enzyme activities of rhizosphere and bulk soils at different salinities. Biol Fertil Soils. 2011;47: 543-554.

69. Nath M, Bhatt D, Prasad R, Tuteja N. Reactive Oxygen Species (ROS) Metabolism and Signaling in Plant-Mycorrhizal Association Under Biotic and Abiotic Stress Conditions. In: Varma A, Prasad R, Tuteja N, editors. Mycorrhiza - Eco-Physiology, Secondary Metabolites, Nanomaterials. Cham: Springer International Publishing; 2017. pp. 223-232.

70. Nishizawa A, Yabuta Y, Shigeoka S. Galactinol and raffinose constitute a novel function to protect plants from oxidative damage. Plant Physiol. 2008;147: 1251-1263.

71. Tognetti VB, Mühlenbock P, Van Breusegem F. Stress homeostasis - the redox and auxin perspective. Plant Cell Environ. 2012;35: 321-333.

72. Tognetti VB, Van Aken O, Morreel K, Vandenbroucke K, van de Cotte B, De Clercq I, et al. Perturbation of indole-3-butyric acid homeostasis by the UDP-glucosyltransferase UGT74E2 modulates Arabidopsis architecture and water stress tolerance. Plant Cell. 2010;22: 2660-2679.

73. Yuan P, Jauregui E, Du L, Tanaka K, Poovaiah BW. Calcium signatures and signaling events orchestrate plant-microbe interactions. Curr Opin Plant Biol. 2017;38: 173-183.

74. McCormack E, Braam J. Calmodulins and related potential calcium sensors of Arabidopsis. New Phytol. 2003;159: 585-598.

75. Cho K-M, Nguyen HTK, Kim SY, Shin JS, Cho DH, Hong SB, et al. CML10, a variant of calmodulin, modulates ascorbic acid synthesis. New Phytol. 2016;209: 664-678.

76. Kazan K, Manners JM. Linking development to defense: auxin in plant-pathogen interactions. Trends Plant Sci. 2009;14: 373-382. album in apple and on growth of the fungus in culture. Can J Plant Sci. 1968;48: 557-559.

78. El-Kazzaz MK. Ethylene effects on in vitro and in vivo growth of certain postharvest fruitinfecting fungi. Phytopathology. 1983;73: 998.

1143 79. KępczyńAska E. Ethylene requirement during germination of Botrytis cinerea spores. 
1144 Physiol Plant. 1989. Available: https://onlinelibrary.wiley.com/doi/abs/10.1111/j.1399-

1145 3054.1989.tb05655.x?casa_token=CrP109f2E9wAAAAA:_yZRIIFQZFXBs89M7po6vo73Dy

1146 8iWNZ7NLCG_yACcW2bxigGLch4fK3tdIS0xbK08ry0vrODnBkcKQw

1147 80. Gryndler M, Hršelová H, Chvátalová I, Jansa J. The effect of selected plant hormones on in 1148 vitro proliferation of hyphae of Glomus fistulosum. Biol Plant. 1998;41: 255-263.

1149 81. Pozo MJ, López-Ráez JA, Azcón-Aguilar C, García-Garrido JM. Phytohormones as 1150 integrators of environmental signals in the regulation of mycorrhizal symbioses. New $1151 \quad$ Phytol. 2015;205: 1431-1436. 


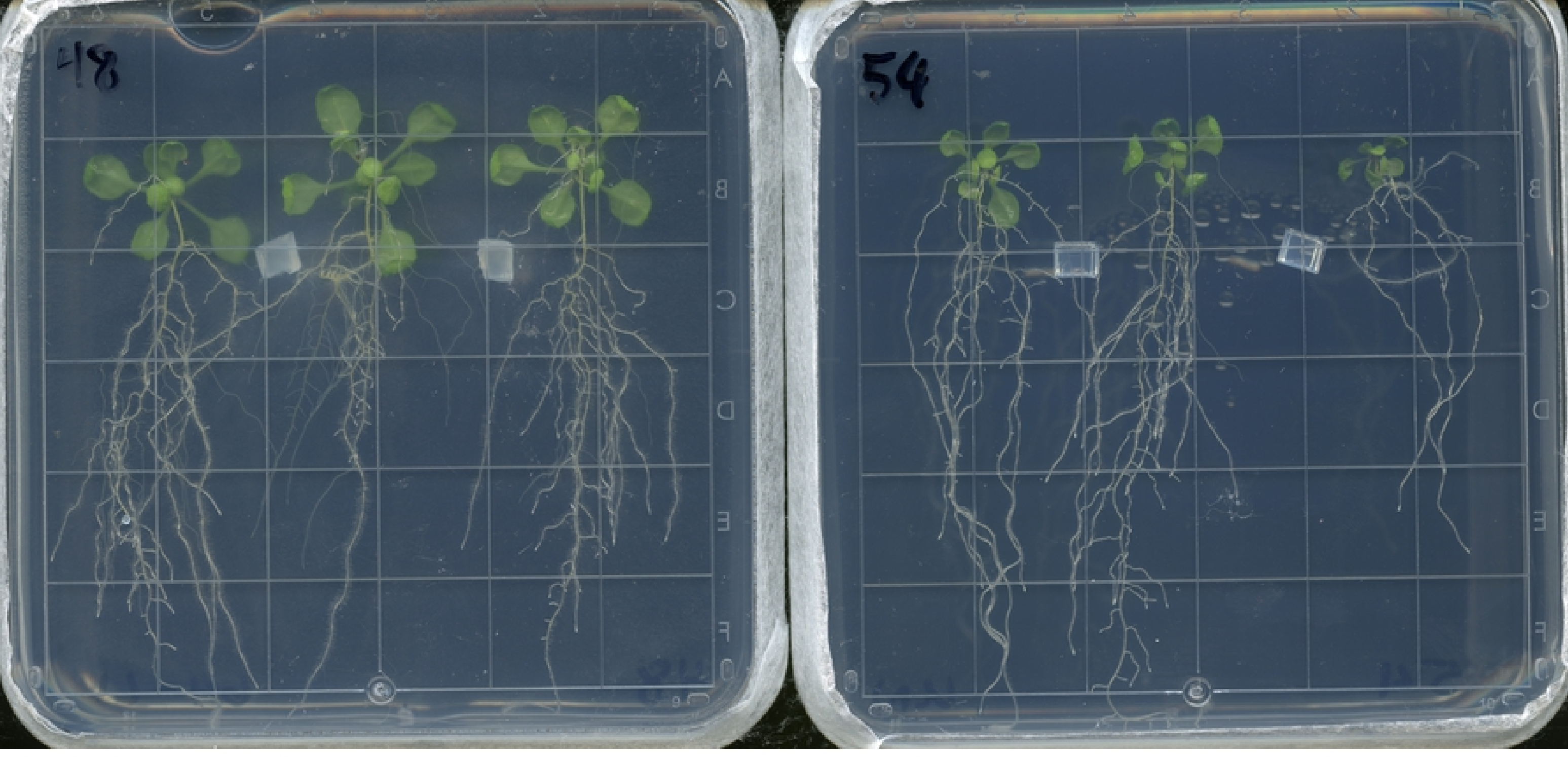

Figure 1 


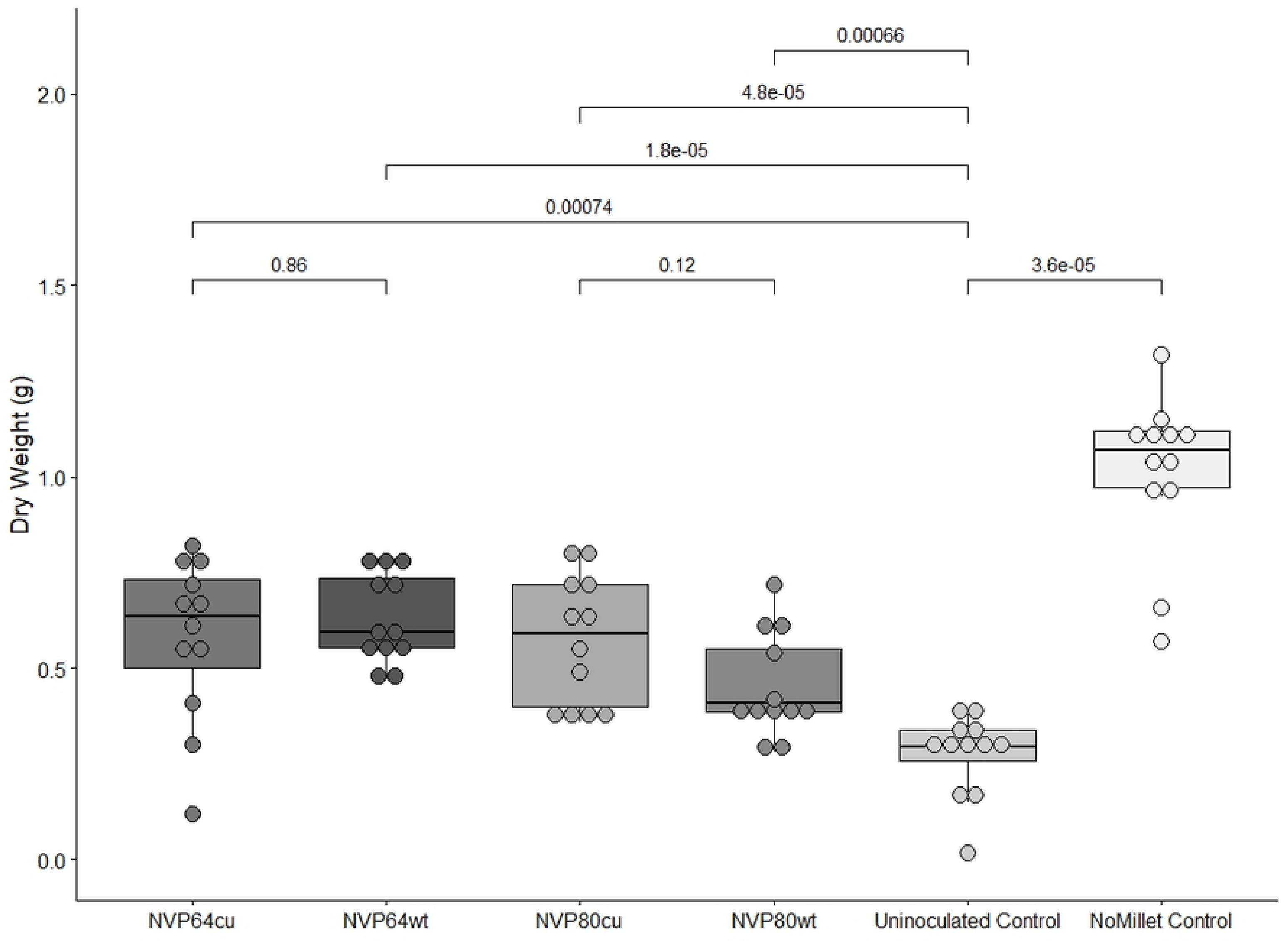

Figure 2 


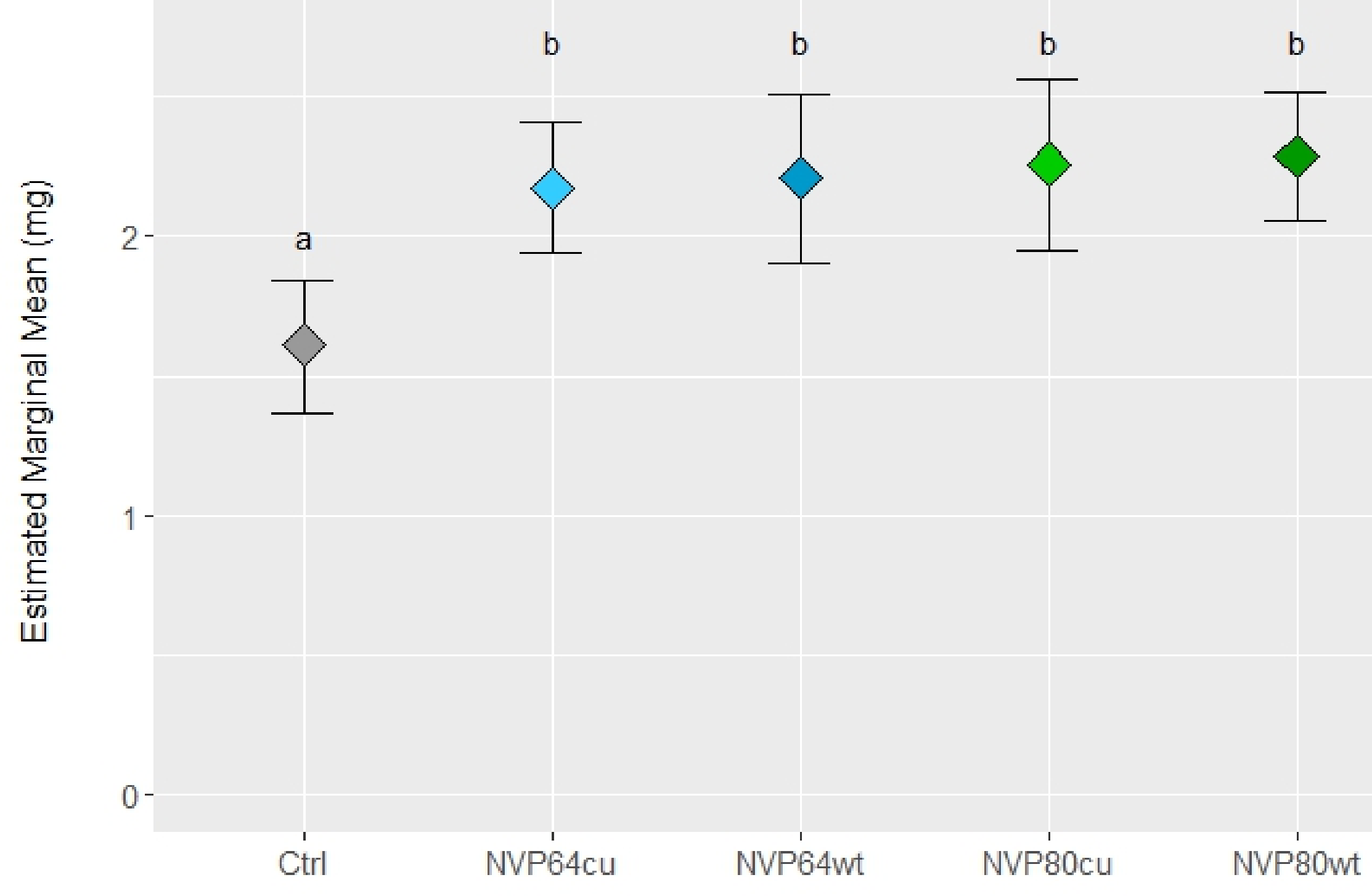

Figure 4 
Figure 7

a) cellular response to hypoxia.

\section{cellular response to decreased oxygen levels}

cellular response to oxygen levels

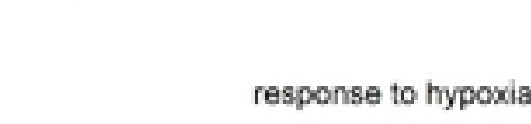

response to decreased oxygen levels

response to oxygen levels

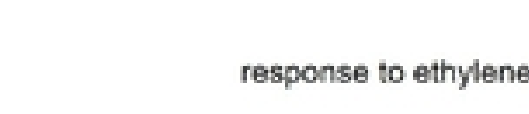

cellular response to ethylene stimulus

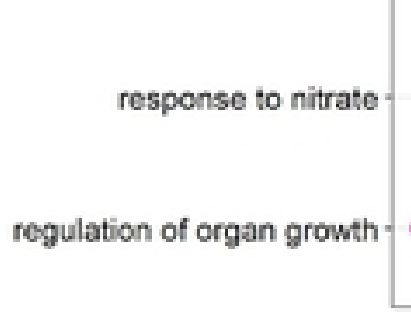

b)

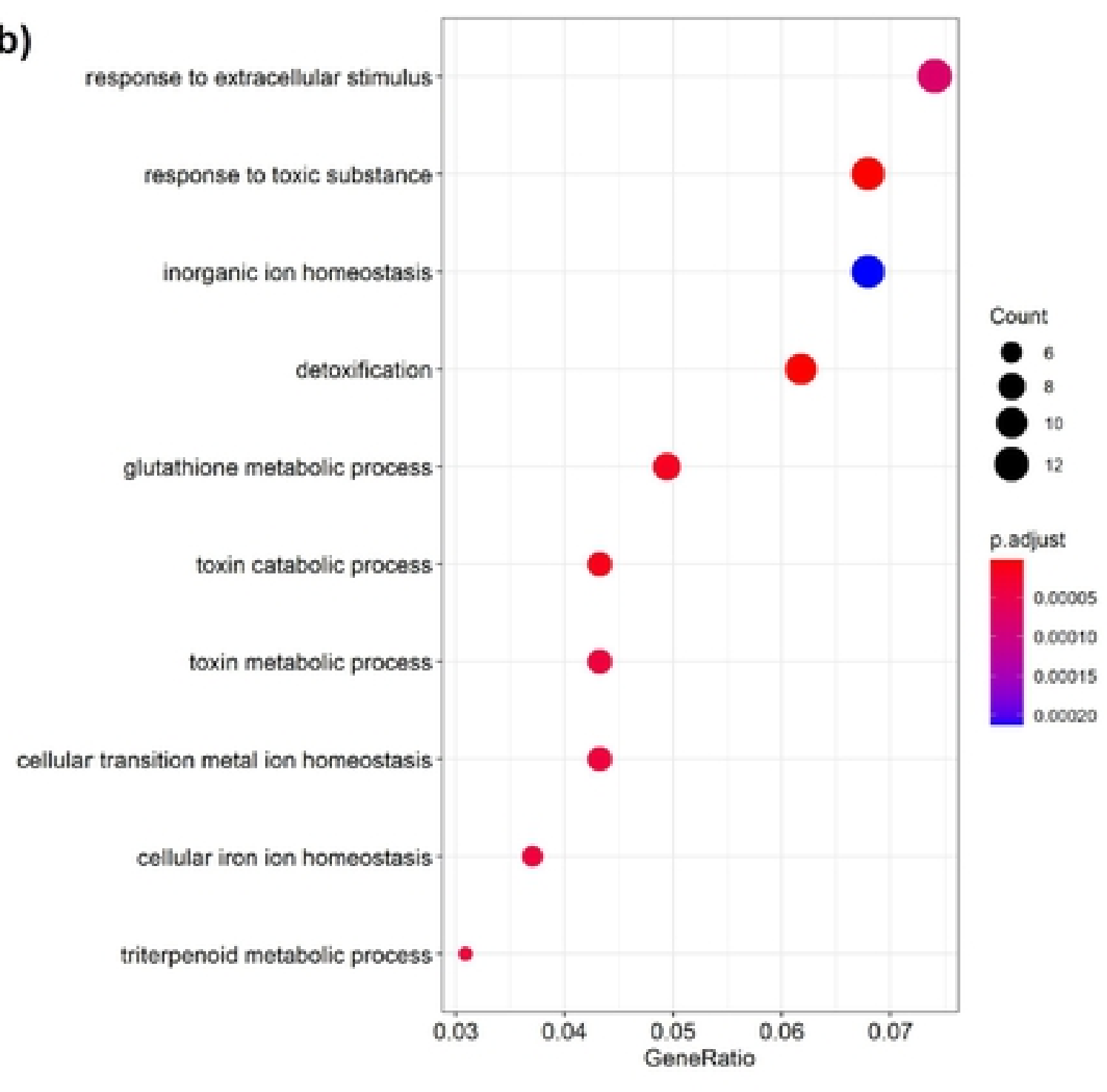


Fixed Effects

\begin{tabular}{|c|c|c|c|c|c|}
\hline & Estimate & |Std.Error & $\overline{\text { df }}$ & $\mid t$-value & $\bar{p}$ \\
\hline (Intercept) & 0.581 & 0.098 & 8.502 & 5.92 & $2.7 \mathrm{E}-04$ \\
\hline reatment=NVP64cu & 0.601 & 0.068 & 230.9 & 8.79 & $3 \mathrm{E}-16$ \\
\hline$t=N V P 64 w t$ & 0.565 & 0.076 & 232.6 & 7.41 & $2 \mathrm{E}-12$ \\
\hline treatrent $=$ NVP $80^{\circ}$ & $0.650^{\text {aiv }}$ & 0.068 & 230.8 & 9.52 & $<2 \mathrm{E}-16$ \\
\hline treatment=NVP80wt & 0.681 & 0.076 & 231.9 & 8.93 & $<2 \mathrm{E}-16$ \\
\hline Root Length & 0.122 & 0.008 & $\overline{514.1}$ & 16.06 & $<2 \mathrm{E}-16$ \\
\hline
\end{tabular}

\section{Random effects}

\begin{tabular}{|l|c|c|c|c|}
\hline & Name & Variance & Std.Dev. & \# of Groups \\
\hline Plate & (Intercept) & 0.074 & 0.273 & 255 \\
\hline Experiment & (Intercept) & 0.005 & 0.072 & 2 \\
\hline Residual & 0.117 & 0.342 & - & - \\
\hline
\end{tabular}

EMM Pairwise Comparisons

\begin{tabular}{|l|c|c|c|c|c|}
\hline Contrast & estimate & SE & df & t.ratio & $p$ \\
\hline Control - NVP64cu & -0.6005 & 0.069 & 250 & -8.7 & $<.0001$ \\
\hline Control - NVP64wt & -0.5654 & 0.0763 & 249 & -7.41 & $<.0001$ \\
\hline Control - NVP80cu & -0.6498 & 0.0689 & 249 & -9.43 & $<.0001$ \\
\hline Control - NVP80wt & -0.6807 & 0.0762 & 248 & -8.93 & $<.0001$ \\
\hline NVP64cu - NVP64wt & 0.0351 & 0.0689 & 250 & 0.509 & 0.9864 \\
\hline NVP64cu - NVP80cu & -0.0494 & 0.0573 & 249 & -0.86 & 0.9108 \\
\hline NVP64cu - NVP80wt & -0.0803 & 0.069 & 250 & -1.16 & 0.7717 \\
\hline NVP64wt - NVP80cu & -0.0844 & 0.0689 & 250 & -1.23 & 0.7363 \\
\hline NVP64wt - NVP80wt & -0.1153 & 0.0763 & 249 & -1.51 & 0.5555 \\
\hline NVP80cu - NVP80wt & -0.0309 & 0.0689 & 249 & -0.45 & 0.9916 \\
\hline
\end{tabular}




\begin{tabular}{|c|c|c|c|c|c|c|c|c|}
\hline \multicolumn{3}{|r|}{ Functional Annotation } & \multicolumn{4}{|c|}{ Log2 Fold-Change } & \multirow{2}{*}{ Name } & \multirow{2}{*}{ Gene } \\
\hline Broad & Middle & Detail & NVP & NVP & NVP & NVP & & \\
\hline \multirow{2}{*}{ Abiotic Stress } & \multirow{2}{*}{\begin{tabular}{|l|} 
Hypoxia/ \\
Oxidative Stress
\end{tabular}} & Peroxidase superfamily protein & 2.26 & 1.92 & 1.84 & 1.98 & PER28 & AT3G03670 \\
\hline & & Stachyose synthase, Raffinose synthase 4 & 1.72 & 0.93 & 1.31 & 1.54 & STS & AT4G01970 \\
\hline \multirow{6}{*}{ Defense } & \multirow{2}{*}{ Bacteria } & Leucine-rich receptor-like protein kinase family protein & 1.5 & & 0.94 & 1.18 & FLS2 & AT5G46330 \\
\hline & & Calcium-binding EF hand family protein & 1.38 & 1.57 & 1.78 & 1.63 & CML12 & AT2G41100 \\
\hline & \multirow{4}{*}{ Fungus } & Chitinase family protein & 3.02 & & 1.98 & 2.36 & F18019.27 & AT2G43620 \\
\hline & & homolog of RPWB 3 & 1.08 & & 1.1 & 1.04 & HR3 & AT3G50470 \\
\hline & & SBP (S-ribonuclease binding protein) family protein & -4.15 & -3.1 & -3.2 & -3.49 & d $48875 \mathrm{c}$ & AT4G17680 \\
\hline & & 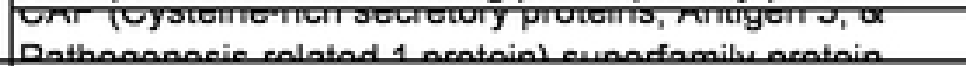 & 2.77 & 2.17 & 3.08 & 2.63 & CAPE3 & AT4G33720 \\
\hline \multirow{6}{*}{ Development } & \multirow{2}{*}{ Growth } & promotes cell growth in response to light & 0.96 & 0.7 & 1.16 & 0.77 & LSH10 & AT2G42610 \\
\hline & & xanthine dehydrogenase 2 & 0.82 & 1 & 0.91 & 0.75 & $\mathrm{XDH} 2$ & AT4G34900 \\
\hline & \multirow{4}{*}{ Root } & 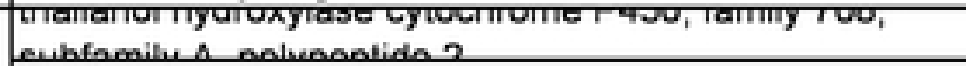 & -1.16 & & & -1.19 & THAH & AT5G48000 \\
\hline & & Thalianol synthase 1 & -1.93 & & & -1.71 & THAS & AT5G48010 \\
\hline & & marneral oxidase & -0.76 & -1.05 & -0.87 & -0.92 & MRO & AT5G42590 \\
\hline & & 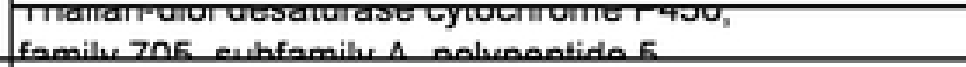 & -1.65 & -1.38 & -1.42 & -1.64 & THAD1 & AT5G47990 \\
\hline \multirow{11}{*}{$\begin{array}{l}\text { Hormone } \\
\text { Signaling }\end{array}$} & \multirow{2}{*}{ Auxin } & Nitrilase 1 & -0.77 & & & -0.78 & NIT1 & AT3G44310 \\
\hline & & nitrilase 2 & -1.05 & & -0.8 & -0.87 & NIT2 & AT3G44300 \\
\hline & \multirow{2}{*}{ Brassinosteroid } & squalene monooxygenase 2 & -1.8 & -1.76 & & -2.13 & SQE4 & AT5G24140 \\
\hline & & baruol synthase 1 & 3.8 & & 2.74 & 3.44 & BARS1 & AT4G15370 \\
\hline & Eth/JA & ethylene response factor & 0.74 & 0.76 & 0.69 & 0.8 & ERF59 & AT1G06160 \\
\hline & & Integrase-type DNA-binding superfamily protein & 1.94 & & & 2.04 & TDR1 & AT3G23230 \\
\hline & & ethylene-activated signaling pathway & 1.49 & & & 1.44 & RAP2.9 & AT4G06746 \\
\hline & & ETHYLENE RESPONSE 2 & 0.84 & & 0.79 & 0.84 & ERT2 & AT3G23150 \\
\hline & & 1-amino-cyclopropane-1-carboxylate (ACC) synthase 7 & -1.01 & -0.99 & -0.85 & -1.08 & $\mathrm{ACS} 7$ & AT4G26200 \\
\hline & \multirow{2}{*}{ Signaling } & cell wall-associated kinase & & 2.86 & 3.14 & & WAK1 & AT1G21250 \\
\hline & & wall-associated kinase 2 & 1.41 & 1.11 & 1.53 & 1.16 & WAK2 & AT1G21270 \\
\hline
\end{tabular}

\section{Table 2}




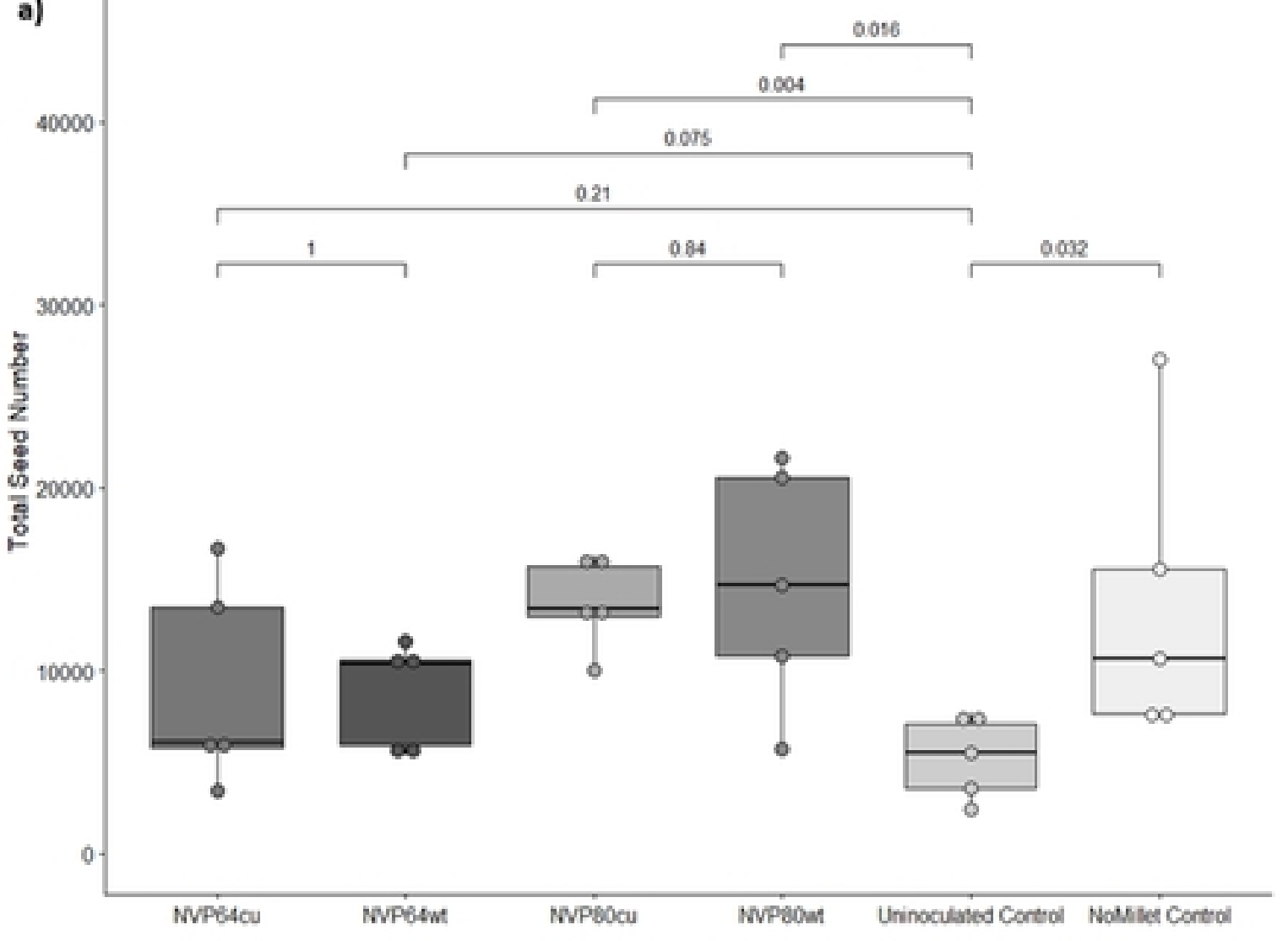

b)

Figure 3
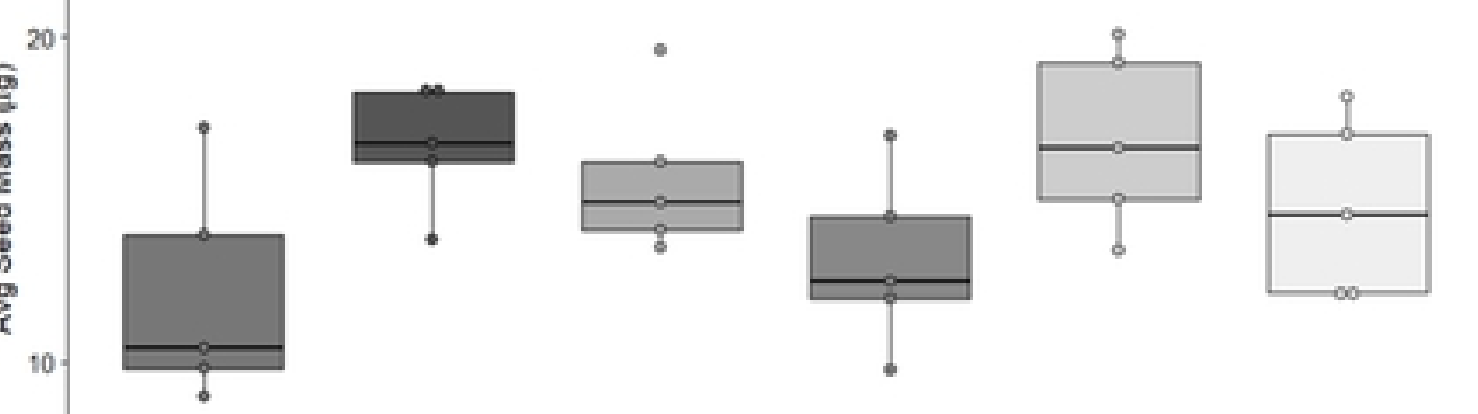
a)

\section{NVP64wt}

NVP80cu

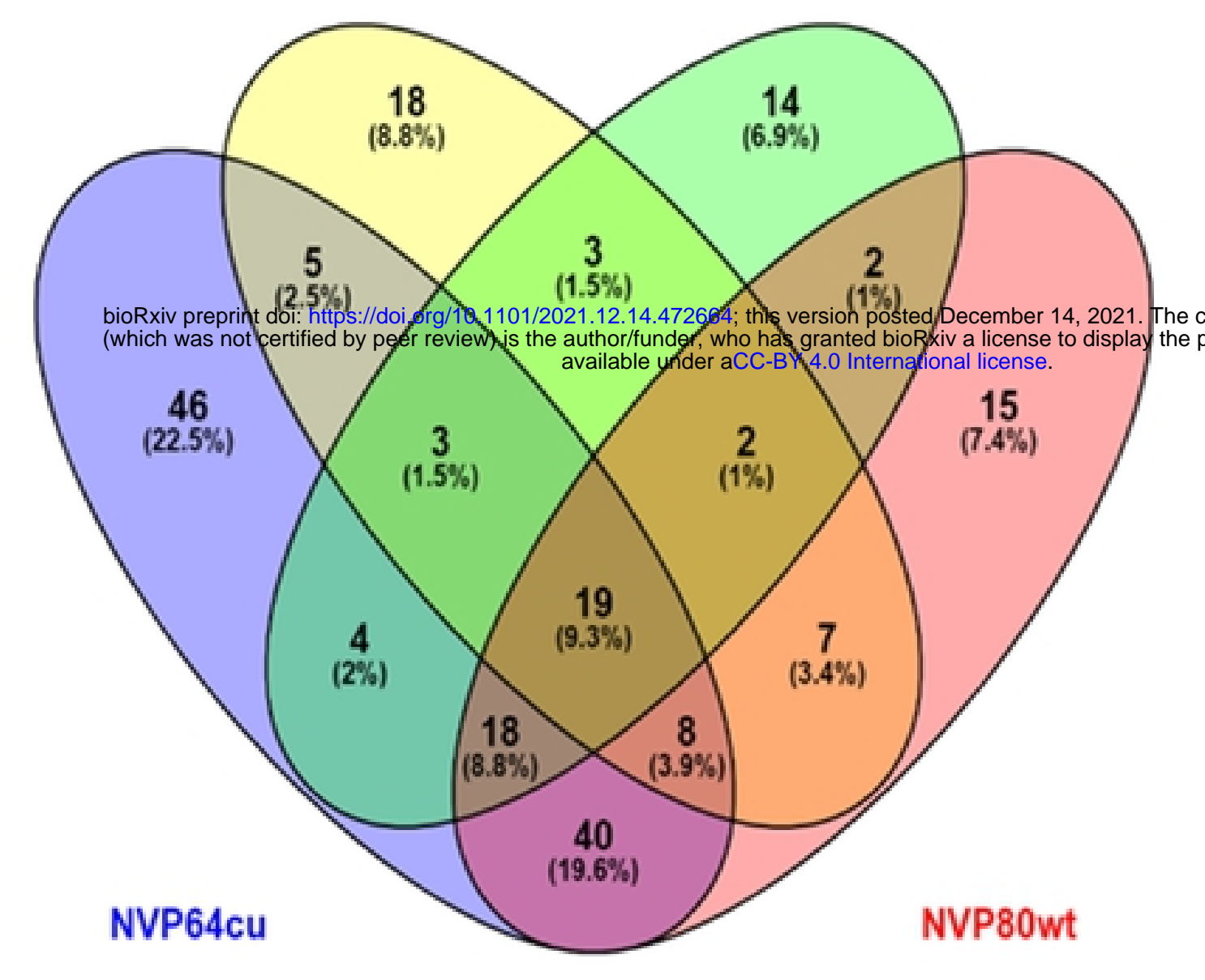

c)

NVP64wt

NVP80cu

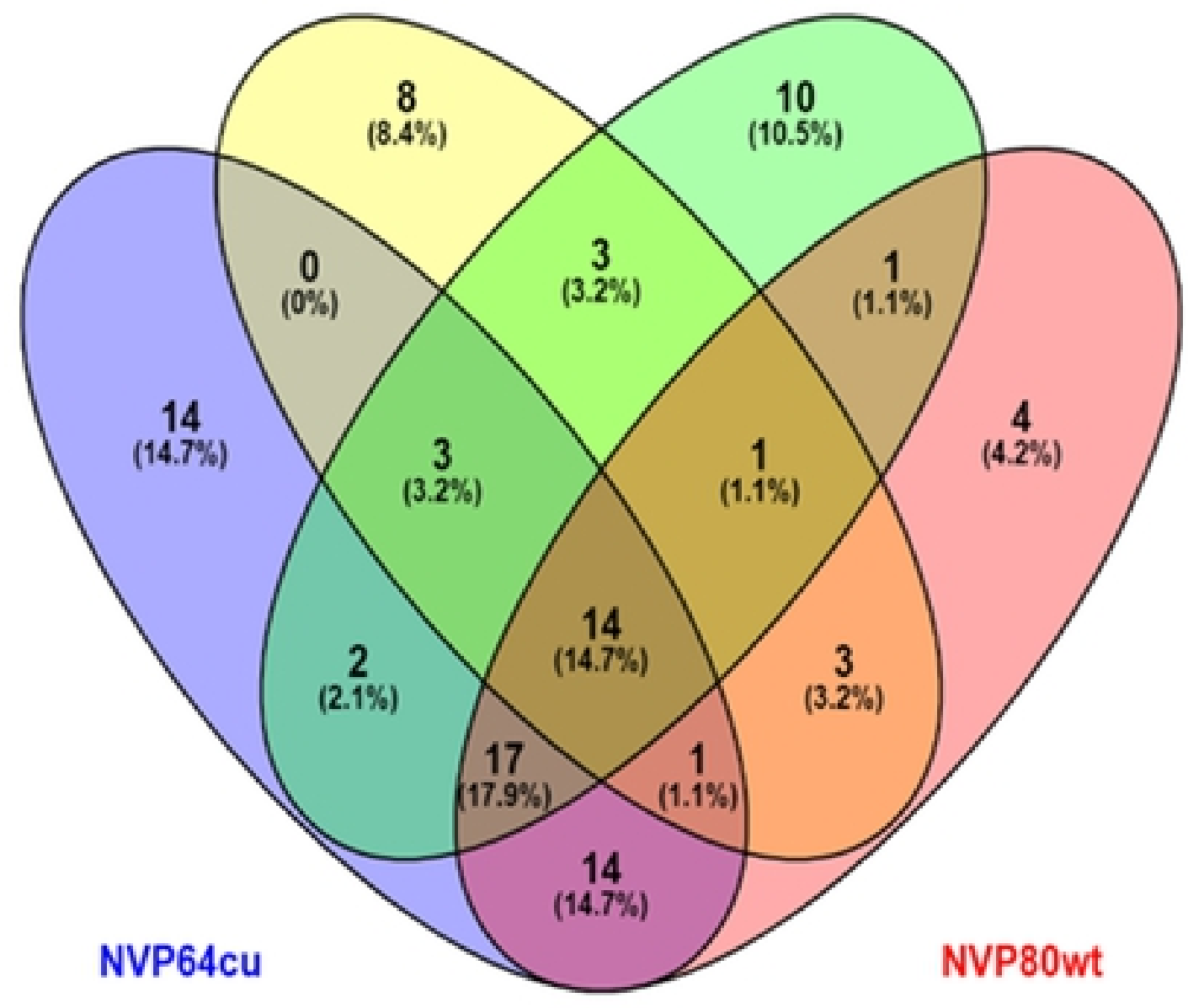

d)

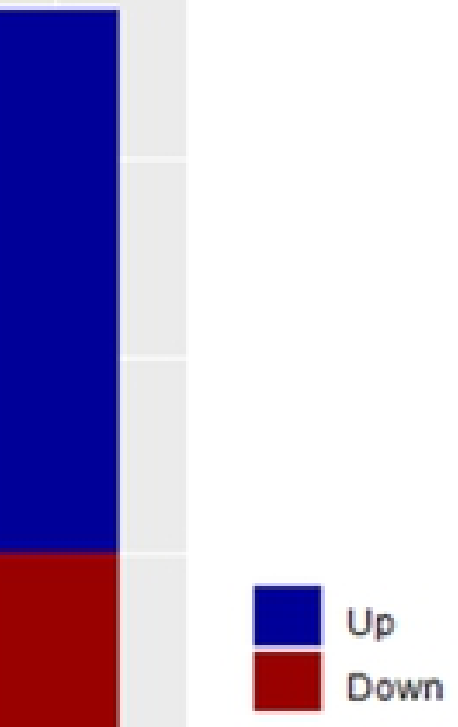

NVP64cu NVP'64wt NVP'80cu NVP'80wt

Treatment

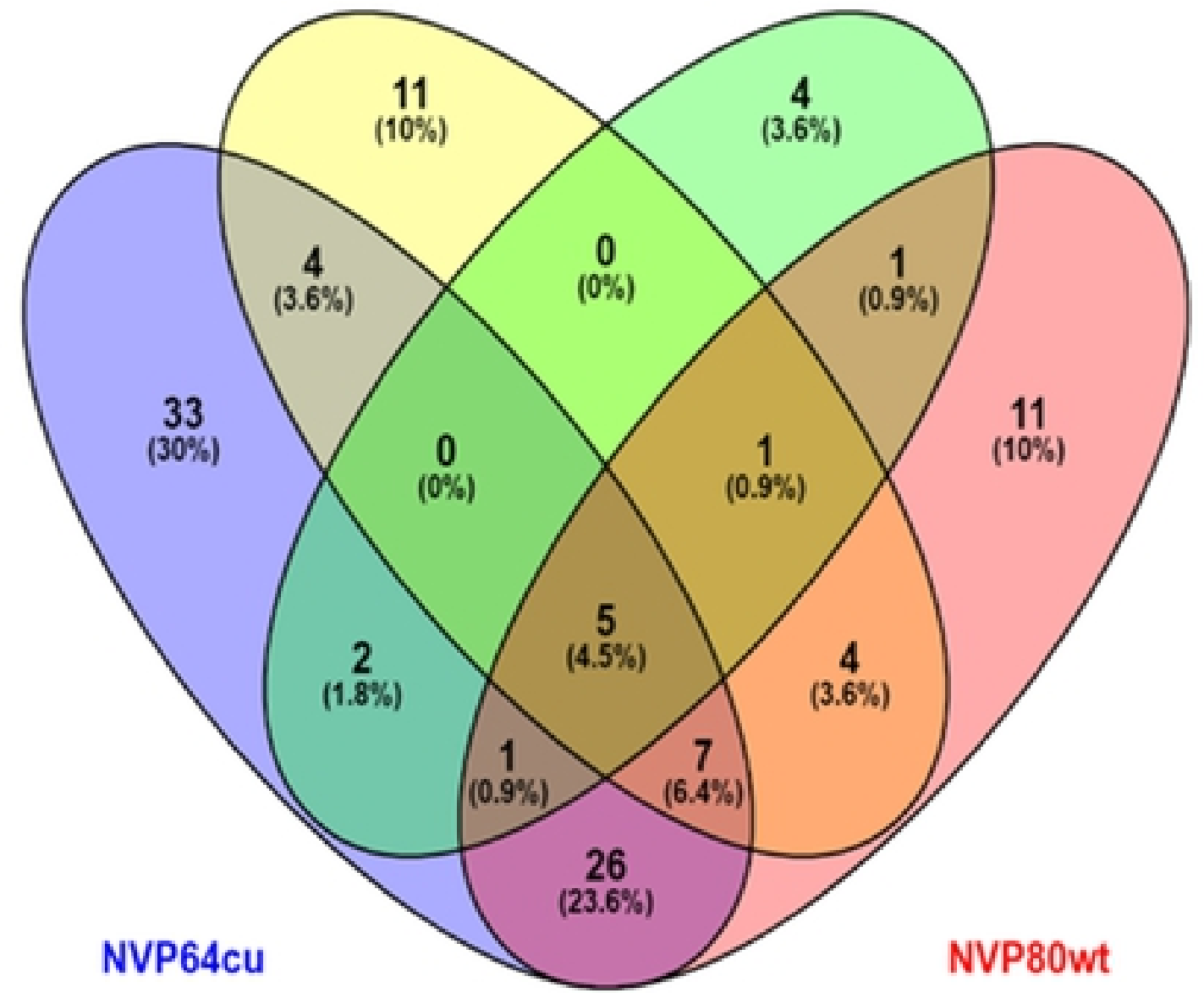

\section{Figure 6}

